\title{
Complementary Kumaraswamy Weibull Power Series Distribution: Some Properties and Application
}

\author{
Innocent Boyle Eraikhuemen, Julian Ibezimako Mbegbu and Friday Ewere
}

\begin{abstract}
In this paper, we propose Complementary Kumaraswamy Weibull Power Series (CKWPS) Distributions. The method is obtained by compounding the Kumaraswamy-G distribution and Power Series distribution on a latent complementary distance problem base. The mathematical properties of the proposed class of distribution are studied. The method of Maximum Likelihood Estimation is used for obtaining the estimates of the model parameters. A member of the family is investigated in detail. Finally an application of the proposed class is illustrated using a real data set.
\end{abstract}

\section{Introduction}

In recent times, several compound models have been developed by complementary risk motivation and applied in several areas. Complementary risk problems arise in several areas, such as engineering, public health, economics actuarial science, biomedical studies, demography and industrial reliability.

The event of interest in latent complementary risk scenarios is related to causes which are not completely observable; rather we observe only the maximum lifetime value among all risks. Since it is not possible to observe the lifetime of the event of interest, the event of interest is modeled as a function of the available information, which is taken as the maximum ordered lifetime value among all causes. Example of industrial applications shows that, the failure of a system can be due to several competing causes such as error in design, contamination from dirt and failure of a component, an assembly

Received: May 8, 2020; Accepted: June 15, 2020

2010 Mathematics Subject Classification: 62P05, 62P10, 62P20.

Keywords and phrases: Kumaraswamy Weibull distribution, power series distribution, latent complementary risk, maximum likelihood. 
error and harsh working environments. Furthermore, in medical applications, the death of a patient can be due to several competing causes such as heart failure, malaria, cancer, diabetes, dementias, tuberculosis and stroke, among others.

In probability modeling, several researchers proposed different approaches of mixing distributions in order to establish more flexible distributions. Some of these distributions were generated by mixing two or more continuous distributions [1], while others by mixing continuous and discrete probability distributions ([2], [3], [4]). Furthermore, other authors derived new compounding distributions by mixing continuous distributions with power series distributions. [5] proposed the compounding of Weibull and power series distributions, [6] generalized exponential Power series distributions, [7] proposed the compound class of extended Weibull power series distributions. [8] introduced the Burr XII power series distributions, [9] introduced the Lindley power series class of distributions.

In this paper, we consider the mixing of continuous and discrete probability distributions. These families of distributions provide some flexibility in modeling data in practical applications; most of the distributions generated by this procedure are obtained by either compounding the minimum or maximum failure rate of the random variable distributed according to the continuous distribution under consideration.

Specifically, a new lifetime family of distributions is proposed by compounding the Kumaraswamy-G family and power series family of distributions. The resulting distribution is called the Complementary Kumaraswamy-G Power Series (CK-GPS) distribution.

Various statistical properties of the proposed distribution along with its reliability features are explored and characterizations are given.

\section{The Complementary Kumaraswamy Weibull Power Series (CKWPS) Distribution}

In the classical complementary risk scenarios, it is impossible to observe the lifetime associated with a particular risk, rather we observe only the maximum lifetime value among all risk. This implies that only component lifetime of a parallel system is observed. The basic idea of introducing the CKWPS is that a lifetime of a system with $N$ (discrete random variable) components and the positive continuous random variable, say 
$X_{i}$ (the lifetime of $i^{\text {th }}$ component), can be denoted by the non-negative random variable $Y=\min \left(X_{1}+X_{2}+\cdots+X_{N}\right)$ or $Y=\max \left(X_{1}+X_{2}+\cdots+X_{N}\right)$, based on whether the components are series or parallel.

Here, a new lifetime family of distributions is proposed by compounding the Kumaraswamy Weibull family and power series family of distributions. The resulting distribution is called the Complementary Kumaraswamy Weibull Power Series (CKWPS) distribution as a complementary to the Kumaraswamy Weibull Power Series (KWPS) distribution by considering the maximum failure rate of independent and identically random variables. The time to the last failure can be appropriately modeled by the Complementary Kumaraswamy Weibull Power Series (CKWPS) class of distribution.

One of the most important distributions used in modeling lifetime data is the Weibull distribution, whose cumulative distribution function (cdf) and probability density function (pdf) are respectively given by

$$
G(x)=1-\exp (-\alpha x)^{\beta}, \quad x, \alpha, \beta>0
$$

and

$$
g(x)=\alpha \beta x^{\beta-1} \exp (-\alpha x)^{\beta}, \quad x, \alpha, \beta>0
$$

where $\alpha$ is a scale parameter and $\beta$ is a shape parameter.

[10] proposed a generalized class of distribution called the Kumaraswamy Generated (K-G) distribution which has a cdf given by

$$
Q_{a, b}(x)=1-\left[1-G(x)^{a}\right]^{b}, \quad x, a, b>0
$$

and the corresponding (pdf) given by

$$
q_{a, b}(x)=\operatorname{abg}(x) G(x)^{a-1}\left[1-G(x)^{a}\right]^{b-1}, \quad x, a, b>0
$$

where $a$ and $b$ are additional shape parameters.

The cdf of the Kumaraswamy Weibull distribution is given as:

$$
F(x)=1-\left(1-\left[1-\exp \left\{-(\alpha x)^{\beta}\right\}\right]^{a}\right)^{b}, \quad x, a, b, \alpha, \beta>0
$$


and the corresponding pdf of Kumaraswamy Weibull distribution is given as:

$$
f(x)=a b \alpha \beta \alpha^{\beta} x^{\beta-1} \exp -(\alpha x)^{\beta}\left[1-\exp -(\alpha x)^{\beta}\right]^{a-1}\left(1-\left[1-\exp \left\{-(\alpha x)^{\beta}\right\}\right]^{a}\right)^{b-1} .
$$

The Kumaraswamy Weibull Power Series (KWPS) models are obtained by compounding the Kumaraswamy Weibull and Power Series distributions. The compounding procedure follows the same set-up pioneered by [11].

The power series family of discrete univariate distributions is credited to [12] even though the earliest work on this family of distributions is due to [13]. Let $N$ be a discrete random variable having a power series distribution with probability mass function (pmf) given by

$$
P(M=m)=\frac{c_{m} \theta^{m}}{D(\theta)}, \quad m=1,2, \ldots
$$

where $C_{m} \geq 0$ depends only on $m, D(\theta)=\sum_{m=1}^{\infty} C_{m} \theta^{m}$ and $\theta>0$ is such that $D(\theta)$ is finite and its first, second and third derivatives exist and are defined by $D^{\prime}(\theta), D^{\prime \prime}(\theta)$ and $D^{\prime \prime \prime}(\theta)$ respectively. [14], [15] have explored the various properties of the power series family of distributions.

Some members of the Power Series family of distributions alongside their respective $c_{m}, D(\theta), D^{\prime}(\theta), D^{\prime \prime}(\theta)$ and $D^{\prime \prime \prime}(\theta)$ are shown in Table 1 ([12] and [13]), where $m$ is the number of trials that resulted in $n$ success.

Table 1. Useful quantities for some power series distributions.

\begin{tabular}{|c|c|c|c|c|c|c|c|}
\hline Distribution & $c_{n}$ & $D(\theta)$ & $D^{\prime}(\theta)$ & $D^{\prime \prime}(\theta)$ & $D^{\prime \prime \prime}(\theta)$ & $D(\theta)^{-1}$ & $\begin{array}{c}\text { Parameter } \\
\text { space }\end{array}$ \\
\hline Possion & $(n !)^{-1}$ & $e^{\theta}-1$ & $e^{\theta}$ & $e^{\theta}$ & $e^{\theta}$ & $\log (\theta+1)$ & $\theta \in(0, \infty)$ \\
\hline Geometric & 1 & $\theta(1-\theta)^{-1}$ & $(1-\theta)^{-2}$ & $2(1-\theta)^{-3}$ & $6(1-\theta)^{-4}$ & $\theta(\theta+1)^{-1}$ & $\theta \in(0,1)$ \\
\hline Logarithmic & $(n)^{-1}$ & $-\log (1-\theta)$ & $(1-\theta)^{-1}$ & $(1-\theta)^{-2}$ & $2(1-\theta)^{-3}$ & $1-e^{-\theta}$ & $\theta \in(0,1)$ \\
\hline Binomial & $\left(\begin{array}{c}m \\
n\end{array}\right)$ & $(\theta+1)^{m}-1$ & $m(1+\theta)^{m-1}$ & $\frac{m(m-1)}{(\theta+1)^{2-m}}$ & $\frac{m(m-1)(m-2)}{(\theta+1)^{2-m}}$ & $(\theta-1)^{1 / m}-1$ & $\theta \in(0, \infty)$ \\
\hline
\end{tabular}


Now, let $X_{1}, X_{2}, \ldots, X_{M}$ be independent and identically distributed (iid) random variable of size $m$ from the Kumaraswamy-G family of distributions as defined in (2.3) and (2.4). Suppose $N$ is discrete and follows the power series distribution in (2.7), then the cdf and pdf of the Complementary Kumaraswamy-G Power Series (CK-GPS) class of distribution are given by

$$
\begin{aligned}
F_{C K G P S}(x) & =\frac{1}{D(\theta)} \sum_{m=i}^{\infty} c_{m}\left(\theta\left\{1-\left(1-G(x)^{a}\right)^{b}\right\}\right)^{m} \\
F_{C K G P S}(x) & =\frac{D\left[\theta\left(1-\left\{1-G(x)^{a}\right\}^{b}\right)\right]}{D(\theta)}
\end{aligned}
$$

and

$$
f_{C K G P S}(x)=\operatorname{ab\theta g}(x) G(x)^{a-1}[1-G(x)]^{b-1} \frac{1-G(x)\left[\theta\left(1-\left\{1-G(x)^{a}\right\}^{b}\right)\right]}{D(\theta)}
$$

with $G(x)$ as Weibull distribution in (2.9), the cdf and pdf of the proposed CKWPS family of distributions are given by

$$
F_{C K W P S}(x)=\frac{D\left(\theta\left\{1-\left(1-\left[1-\exp \left\{-(\alpha x)^{\beta}\right\}\right]^{a}\right)^{b}\right\}\right)}{D(\theta)}, \quad x, a, b, \alpha, \theta>0
$$

and

$$
\begin{aligned}
f_{C K W P S}(x) & =a b \alpha \beta \theta x^{\beta-1} \exp \left\{-(\alpha x)^{\beta}\right\}\left(1-\exp \left\{-(\alpha x)^{\beta}\right\}\right)^{a-1} \\
& \times\left(1-\left[1-\exp \left\{-(\alpha x)^{\beta}\right\}\right]^{a}\right)^{b-1} \frac{D^{\prime}\left(\theta\left\{1-\left(1-\left[1-\exp \left\{-(\alpha x)^{\beta}\right\}\right]^{a}\right)^{b}\right\}\right)}{D(\theta)}
\end{aligned}
$$

respectively. 


\section{Some Properties of the CKWPS Distribution}

\subsection{The survival and hazard rate functions}

For a continuous distribution function with pdf (2.10) and cdf (2.11), the survival function of the CKWPS is given by

$$
S_{C K W P S}(x)=\frac{D(\theta)-D\left(\theta\left\{1-\left(1-\left[1-\exp \left\{-(\alpha x)^{\beta}\right\}\right]^{a}\right)^{b}\right\}\right)}{D(\theta)}, \quad x, a, b, \alpha, \theta, \beta>0
$$

and the hazard rate function of the CKWPS is given by

$$
h_{C K W P S}(x)=\frac{a b \alpha \beta \theta x^{\beta-1} \exp \left\{-(\alpha x)^{\beta}\right\}\left(1-\exp \left\{-(\alpha x)^{\beta}\right\}\right)^{a-1}\left(1-\left[1-\exp \left\{-(\alpha x)^{\beta}\right\}\right]^{a}\right)^{b-1}}{\times D^{\prime}\left(\theta\left\{1-\left(1-\left[1-\exp \left\{-(\alpha x)^{\beta}\right\}\right]^{a}\right)^{b}\right\}\right)} .
$$

Proposition 3.1. The Kumaraswamy-Weibull class of distribution is a special case of the Complementary Kumaraswamy-Weibull Power Series (CKWPS) class of distributions when $\theta$ approaches $0^{+}$.

Proof. By the argument of [5] and applying the definition $D(\theta)=\sum_{m=1}^{\infty} a_{m} \theta^{m}$, we obtain

$$
F_{C K W-P S}(x)=1-\frac{\sum_{m=1}^{\infty} a_{m}\left[\theta\left\{\left(1-\left[1-\exp \left\{-(\alpha x)^{\beta}\right\}\right]^{a}\right)^{b}\right\}\right]^{m}}{\sum_{m=1}^{\infty} a_{m} \theta^{n}}
$$

As $\theta \rightarrow 0^{+}$, we write 


$$
\lim _{\theta \rightarrow 0^{+}} F_{C K W-P S}(x)=1-\lim _{\theta \rightarrow 0^{+}} \frac{\sum_{m=1}^{\infty} a_{m}\left[\theta\left\{\left(1-\left[1-\exp \left\{-(\alpha x)^{\beta}\right\}\right]^{a}\right)^{b}\right\}\right]^{m}}{\sum_{m=1}^{\infty} c_{m} \theta^{m}} .
$$

Evaluating this limit gives

$$
\begin{gathered}
a_{1}\left\{\left(1-[1-\exp \{-(\alpha x) \beta\}]^{a}\right)^{b}\right\} \\
\lim _{\theta \rightarrow 0^{+}} F_{C K W-P S}(x)=1-\lim _{\theta \rightarrow 0^{+}} \frac{+\sum_{m=2}^{\infty} m a_{m} \theta^{m-1}\left\{\left(1-\left[1-\exp \left\{-(\alpha x)^{\beta}\right\}\right]^{a}\right)^{b}\right\}^{m}}{a_{1}+\sum_{m=2}^{\infty} m c_{m} \theta^{m-1}} \\
=\left\{1-\left(1-\left[1-\exp \left\{-(\alpha x)^{\beta}\right\}\right]^{a}\right)^{b}\right\} .
\end{gathered}
$$

This is the cdf of the Kumaraswamy-Weibull distribution.

Proposition 3.2. The $p d f$ of the Complementary Kumaraswamy-G Power Series family of distributions can be expressed as an infinite linear combination of density of the first order statistics of the Kumaraswamy-G distribution as

$$
f_{C K W P S}(x)=\sum_{n=1}^{\infty} P_{m}(M=m) f_{X_{x_{(m)}}}(x ; m),
$$

where $f_{X_{x_{(m)}}}(x ; m)$ is the pdf of $X_{(m)}=\max \left\{X_{i}\right\}_{i=1}^{m}$ and $P_{m}(M=m)$ is as in (2.7).

Proof. Given that $D^{\prime}(\theta)=\sum_{m=2}^{\infty} m a_{m} \theta^{m-1}$ and from equation (2.11), we have

$$
\begin{gathered}
f_{C K W P S}(x)=a b \alpha \beta \theta x^{\beta-1} \exp \left\{-(\alpha x)^{\beta}\right\}\left[1-\exp \left\{-(\alpha x)^{\beta}\right\}\right]^{a-1}\left(1-\left[1--\exp \left\{-(\alpha x)^{\beta}\right\}\right]^{a}\right)^{b-1} \\
\times \frac{\sum_{m=1}^{\infty} m a_{m} \theta^{m}\left\{1-\left(1-\left[1-\exp \left\{-(\alpha x)^{\beta}\right\}\right]^{a}\right)^{b}\right\}^{m-1}}{D(\theta)}
\end{gathered}
$$




$$
\begin{aligned}
& f_{C K W P S}(x)=\sum_{m=1}^{\infty} \frac{a_{m} \theta^{m}}{D(\theta)} a b \alpha \beta \theta x^{\beta-1} \exp \left\{-(\alpha x)^{\beta}\right\}\left[1-\exp \left\{-(\alpha x)^{\beta}\right\}\right]^{a-1} \\
& \times\left(1-\left[1-\exp \left\{-(\alpha x)^{\beta}\right\}\right]^{a}\right)^{b-1}\left\{1-\left(1-\left[1-\exp \left\{-(\alpha x)^{\beta}\right\}\right]^{a}\right)^{b}\right\}^{m-1} \\
& f_{X_{x_{(m)}}}(x ; m)=m a b \alpha \beta \theta x^{\beta-1} \exp \left\{-(\alpha x)^{\beta}\right\}\left[1-\exp \left\{-(\alpha x)^{\beta}\right\}\right]^{a-1} \\
& \times\left(1-\left[1-\exp \left\{-(\alpha x)^{\beta}\right\}\right]^{a}\right)^{b-1}\left\{1-\left(1-\left[1-\exp \left\{-(\alpha x)^{\beta}\right\}\right]^{a}\right)^{b}\right\}^{m-1} .
\end{aligned}
$$

Hence, this completes the proof

\subsection{Expansion of the CKWPS density function}

Using Proposition 3.2,

$$
f_{C K W P S}(x)=\sum_{m=1}^{\infty} \frac{c_{m} \theta^{m}}{D(\theta)} f_{X_{x_{(m)}}}(x ; m),
$$

where

$$
f_{X_{x(m)}}(x ; m)=m a b \alpha \beta x^{\beta-1} \exp \left\{-(\alpha x)^{\beta}\right\}\left[1-\exp \left\{-(\alpha x)^{\beta}\right\}\right]^{a-1}\left[1-\left(1-\exp \left\{-(\alpha x)^{\beta}\right\}\right)^{a}\right]^{b-1} \text {. }
$$

By the series representation

$$
\begin{aligned}
& (1-z)^{a-1}=\sum_{p=0}^{\infty}(-1)^{j}\left(\begin{array}{c}
a-1 \\
j
\end{array}\right) z^{j}, \quad a>0,|z|<1 \\
& f(x)=\sum_{p=0}^{\infty}(-1)^{j}\left(\begin{array}{c}
b-k \\
j
\end{array}\right) a b g_{w p}(x)\left(G_{w p}(x)\right)^{a j+a-1} \\
& f(x)=\sum_{j=0}^{\infty}\left(\begin{array}{c}
b-1 \\
j
\end{array}\right)(-1)^{j} a b \alpha \beta \theta x^{\beta-1} \exp \left(-\alpha x^{\beta}\right) \frac{\left[\exp \left(-\alpha x^{\beta}\right)\right]}{\exp (\theta)-1}\left(\frac{1-\left[\exp \left(-\alpha x^{\beta}\right)\right]}{\exp (\theta)-1}\right)^{a j+a-1}
\end{aligned}
$$


$=\sum_{j=0}^{\infty}\left(\begin{array}{c}b-1 \\ j\end{array}\right)(-1)^{a j+a+j-1} \alpha \beta \theta a b x^{\beta-1} \exp \left(-\alpha x^{\beta}\right) \frac{\left[\exp \left(-\alpha x^{\beta}\right)\right]}{[\exp (\theta)-1]^{a j+a}}\left(1-\left[\exp \left(-\alpha x^{\beta}\right)\right]\right)^{a j+a-1}$

$=\sum_{j=0}^{\infty} \sum_{k=0}\left(\begin{array}{c}b-1 \\ j\end{array}\right)\left(\begin{array}{c}a j+a-1 \\ k\end{array}\right)(-1)^{a j+a+j-1} \alpha \beta \theta a b x^{\beta-1} \frac{\exp \left(-\alpha x^{\beta}\right)}{[\exp (\theta)-1]^{a j+a}}\left(\left[(k+1) \exp \left(-\alpha x^{\beta}\right)\right]\right)$.

Given that $e^{t}=\sum_{m=0}^{\infty} \frac{t^{m}}{m !}$ applying this to (3.8) gives

$=\sum_{j=0}^{\infty} \sum_{k=0}^{\infty} \sum_{m=0}^{\infty}\left(\begin{array}{c}b-1 \\ j\end{array}\right)\left(\begin{array}{c}a j+a-1 \\ k\end{array}\right) \frac{(-1)^{a j+a+j+k-1}}{[\exp (\theta)-1]^{a j+a}} \frac{a b \theta^{m+1}(k+1)^{m}}{m !} \alpha \beta x^{\beta-1} \exp -(m+1) \alpha x^{\beta}$

$=\sum_{j=0}^{\infty} \sum_{k=0}^{\infty} \sum_{m=0}^{\infty}\left(\begin{array}{c}b-1 \\ j\end{array}\right)\left(\begin{array}{c}a j+a-1 \\ k\end{array}\right) \frac{(-1)^{a j+a+j+k-1}}{[\exp (\theta)-1]^{a j+a}} \frac{a b \theta^{m+1}(k+1)^{m}}{(m+1) !} \alpha \beta x^{\beta-1}(m+1) x^{\beta-1}\left(\exp -\alpha(m+1) x^{\beta}\right)$

$=\sum_{j, k, m=0}^{\infty} w_{j, k, m}(a, b, \theta) g(x ; \alpha[m+1], \beta)$,

where

$$
w_{j, k, m}(a, b, \theta)=\left(\begin{array}{c}
b-1 \\
j
\end{array}\right)\left(\begin{array}{c}
a j+a-1 \\
k
\end{array}\right) \frac{(-1)^{a j+a+j-1}}{[\exp (\theta)-1]^{a j+a}} \frac{a b \theta^{m+1}(k+1)^{m}}{(m+1) !}
$$

are the weights and $g(x ; \alpha[m+1], \beta)$ is the Weibull pdf with scale parameter $\alpha(m+1)$ and shape parameter $\beta$.

\subsection{Quantile function, moments and order statistics of the CKWPS distribution}

Proposition 3.3. The quantile function of the CKWPS distribution is obtained by solving the equation $F(x)=U$, where $F(x)$ is the cdf of the CKWPS distribution. Then

$$
x=\frac{1}{\alpha}\left(-\log \left\{1-\left[1-\left(1-\frac{D^{-1} U D(\theta)}{\theta}\right)^{\frac{1}{b}}\right]^{\frac{1}{a}}\right\}\right)^{\frac{1}{\beta}}
$$

and $D^{-1}(\cdot)$ is the inverse of $D(\cdot)$. 
Proof.

$$
\begin{gathered}
U=\frac{D\left\{\theta\left[1-\left(1-\left[1-\exp \left\{-(\alpha x)^{\beta}\right\}\right]^{a}\right)^{b}\right]\right\}}{D(\theta)} \\
D^{-1} U D(\theta)=\left\{\theta\left[1-\left(1-\left[1-\exp \left\{-(\alpha x)^{\beta}\right\}\right]^{a}\right)^{b}\right]\right\} \\
1-\exp \left\{-(\alpha x)^{\beta}\right\}=\left[1-\left(1-\frac{D^{-1} U D(\theta)}{\theta}\right)^{\frac{1}{b}}\right]^{\frac{1}{a}} \\
x=\frac{1}{\alpha} \int_{\left.-\log \left\{1-\left[1-\left(1-\frac{D^{-1} U D(\theta)}{\theta}\right)^{\frac{1}{b}}\right]^{\frac{1}{a}}\right\}\right)^{\frac{1}{\beta}}}\left([1)^{\frac{1}{\beta}}\right.
\end{gathered}
$$

where $U$ is a uniform random variable on unit interval $(0,1)$.

The median of the CKWPS distribution is obtained by setting $U=0.5$ in (3.11) and obtain

$$
x=\frac{1}{\alpha}\left(-\log \left\{1-\left[1-\left(1-\frac{D^{-1} D(\theta)}{2 \theta}\right)^{\frac{1}{b}}\right]^{\frac{1}{a}}\right)^{\frac{1}{\beta}} .\right.
$$

Proposition 3.4. The $r^{\text {th }}$ moment of a CKWPS distributed random variable $X$ is given by

$$
E\left(X^{r}\right)=\frac{\Gamma(r+1)}{\alpha^{r} D(\theta)} \sum_{m=1}^{\infty} \sum_{j=0}^{m-1} a_{m} \theta^{m} m\left(\begin{array}{c}
m-1 \\
j
\end{array}\right)(-1)^{j} \frac{1}{(j+1)^{r+1}}
$$

Proof. Since $D^{\prime}$ is a non-decreasing function, $D^{\prime}\left(\theta\left\{1-\left(1-[1-\exp (-\alpha x)]^{a}\right)^{b}\right\}\right)$ 
$\leq D^{\prime}(\theta)$. Hence by (2.11) $f(x) \leq \frac{D^{\prime}(\theta)}{D(\theta)} \theta \alpha e^{-\alpha x}$, which implies that $E\left(X^{r}\right)$ exists.

Also $f(x)=\sum_{i=1}^{\infty} \frac{a_{m} \theta^{m}}{D(\theta)} f_{m}(x)$ describes the density of CKWPS as a mixture, then

$$
E\left(X^{r}\right)=\sum_{m=1}^{\infty} \frac{a_{m} \theta^{m}}{D(\theta)} E\left(Y_{m}^{r}\right)
$$

where $Y_{m}$ has $f_{m}$ as its density function, and

$$
E\left(Y_{m}^{r}\right)=\frac{\Gamma(r+1)}{\alpha^{r}} \sum_{j=0}^{m-1} m\left(\begin{array}{c}
m-1 \\
j
\end{array}\right)(-1)^{j} \frac{1}{(j+1)^{r+1}} .
$$

Substituting (3.15) into (3.14) gives equation (3.13).

The first moments about the origin of the CKWPS distribution (the mean) is obtained by setting $r=1$ in (3.13), we have

$$
E\left(x^{r}\right)=\mu_{r}^{\prime}=\frac{\Gamma(2)}{\alpha D(\theta)} \sum_{m=1}^{\infty} \sum_{j=0}^{m-1} a_{m} \theta^{m} m\left(\begin{array}{c}
m-1 \\
j
\end{array}\right)(-1)^{j} \frac{1}{(j+1)^{2}} .
$$

The variance of the CKWPS distribution is given by

$$
\begin{aligned}
\operatorname{Var}(X)= & \sigma^{2}=\mu_{2}^{\prime}-\left(\mu_{1}^{\prime}\right)^{2} \\
= & {\left[\frac{\Gamma(3)}{\alpha^{2} D(\theta)} \sum_{m=1}^{\infty} \sum_{j=0}^{m-1} a_{m} \theta^{m} m\left(\begin{array}{c}
m-1 \\
j
\end{array}\right)(-1)^{j} \frac{1}{(j+1)^{3}}\right] } \\
& -\left[\frac{\Gamma(2)}{\alpha D(\theta)} \sum_{m=1}^{\infty} \sum_{j=0}^{m-1} a_{m} \theta^{m} m\left(\begin{array}{c}
m-1 \\
j
\end{array}\right)(-1)^{j} \frac{1}{(j+1)^{2}}\right]^{2} .
\end{aligned}
$$

The coefficient of skewness of the CKWPS distribution is given by

$$
S k=\frac{E\left[(X-\mu)^{3}\right]}{\left[E(X-\mu)^{2}\right]^{3 / 2}}=\frac{\mu_{3}^{\prime}-3 \mu \mu_{2}^{\prime}+2 \mu^{3}}{\left(\mu_{2}^{\prime}-\mu^{2}\right)^{3 / 2}} .
$$


The coefficient of kurtosis of the CKWPS distribution is given by

$$
K u=\frac{E\left[(X-\mu)^{4}\right]}{\left[E(X-\mu)^{2}\right]^{2}}=\frac{\mu_{4}^{\prime}-4 \mu \mu_{3}^{\prime}+6 \mu^{2} \mu_{2}^{\prime}-3 \mu^{4}}{\left(\mu_{2}^{\prime}-\mu^{2}\right)^{2}}
$$

and the moment generating function of the CKWPS distribution is given as

$$
E\left(e^{t X}\right)=M_{x}(t)=\frac{\Gamma(2)}{\alpha D(\theta)} \sum_{r=0}^{\infty} \sum_{m=1}^{\infty} \sum_{j=0}^{m-1} \frac{t^{r}}{r !} a_{m} \theta^{m} m\left(\begin{array}{c}
m-1 \\
j
\end{array}\right)(-1)^{j} \frac{1}{(j+1)^{2}} .
$$

\section{Order Statistics}

Let the set of observations be ordered as $X_{1}, X_{2}, X_{3}, \ldots, X_{n}$ whereby $X_{1}$ denote the minimum time to failure and $X_{n}$ denote the maximum time to failure. The trials are independent and identically distributed. The pdf of the $k^{\text {th }}$ order statistics from the CKWPS distribution is given as

$$
g_{k: n}(x)=\frac{n ! f(x)}{(k-1) !(n-k) !}[F(x)]^{k-1}[1-F(x)]^{n-1} .
$$

Using the identity $(1-z)^{n-1}=\sum_{p=0}^{\infty}(-1)^{p}\left(\begin{array}{c}n-1 \\ p\end{array}\right) z^{p}$ in (3.21) gives

$$
g_{i ; n}(x)=\frac{n ! f(x)}{(k-1) !(n-k) !} \sum_{p=0}^{\infty}(-1)^{p}\left(\begin{array}{c}
n-k \\
p
\end{array}\right)[F(x)]^{p+k-1} .
$$

Using (2.8) and (2.9) in (3.22), we have

$$
g_{i ; n}(x ; p)=\frac{q(x) D^{\prime}[\theta\{1-(1-G(x))\}]}{B(i, n-i+1)[D(\theta)]^{i+j}} \sum_{j=0}^{n-1}\left(\begin{array}{c}
n-1 \\
j
\end{array}\right)(-1)^{j}\{D[\theta\{1-(1-G(x))\}]\}^{i+j-1},
$$

where $q(x)=\operatorname{abg}(x) G(x)^{a-1}[1-G(x)]^{b-1}, B(.,$.$) denotes the beta function, and$

$$
\begin{aligned}
& \{D[\theta\{1-(1-G(x))\}]]^{i+j-1} \\
= & \left\{\sum_{n=1}^{\infty} a_{n} \theta^{n}\{1-(1-G(x))\}^{n}\right\}^{i+j-1}
\end{aligned}
$$




$$
=a_{1}^{i+j-1} \theta^{i+j-1}\{1-(1-G(x))\}^{i+j-1}\left(\sum_{s+0}^{\infty} k_{s} \theta^{s}\{1-(1-G(x))\}^{s}\right)^{i+j-1},
$$

where $k_{s}=\frac{a_{s+1}}{a_{1}}, s=1,2,3, \ldots$

$$
\begin{aligned}
& \text { Using the identity }\left(\sum_{s=0}^{\infty} k_{s} z^{s}\right)^{j}=\sum d_{j: s} z^{s} \text { for positive integer } j \text {, we have } \\
& D[\theta\{1-(1-G(x))\}]^{i+j-1}=\sum_{s=0}^{\infty} d_{i+j-1 ; s} a^{i+j-1} \theta^{i+j+s-1}\{1-(1-G(x))\}^{l+j+s-1} .
\end{aligned}
$$

By the expansion

$$
\begin{aligned}
& D^{\prime}(\theta)=\sum n a_{n} \theta^{n-1}, \quad \theta>0, \\
& D^{\prime}[\theta\{1-(1-G(x))\}]=a_{1} \sum_{k=0}^{\infty}(k+1) b_{k} \theta^{k}\{1-(1-G(x))\}^{k} .
\end{aligned}
$$

Substituting (3.25) and (3.26) into (3.23), we have

$$
\begin{aligned}
g_{i ; n}(x ; p)= & \frac{q(x) \sum_{k=1}^{\infty} k a_{k} \theta^{k-1}\{1-(1-G(x))\}^{k-1}}{B(i, n-i+1)[D(\theta)]^{i+j}} \\
& \cdot \sum_{j=0}^{n-1}\left(\begin{array}{c}
n-1 \\
j
\end{array}\right)(-1)^{j} \sum_{s=0}^{\infty} d_{i+j-1 ; s} a^{i+j-1} \theta^{i+j+s-1}\{1-(1-G(x))\}^{l+j+s-1} \\
g_{i ; n}(x ; p)= & \frac{q(x)}{B(i, n-i+1) D(\theta)^{t+j}} \cdot \sum_{j=0}^{n-1} \sum_{s=0}^{\infty} \sum_{k=0}^{\infty}\left(\begin{array}{c}
n-1 \\
j
\end{array}\right)(-1)^{j} b_{k}(k+1) \\
& \times d_{i+j-1 ; s} a^{i+j} \theta^{i+j+s+k}\{1-(1-G(x))\}^{(l+j+s+k)} \\
g_{i ; n}(x ; p)= & \sum_{j=0}^{n-1} \sum_{s=0}^{\infty} \sum_{k=0}^{\infty} w_{i, j, s, k}\{1-(1-G(x))\}^{(l+j+s+k)}
\end{aligned}
$$


where

$$
w_{i, j, s, k}=\frac{q(x)\left(\begin{array}{c}
n-1 \\
j
\end{array}\right)(-1)^{j} b_{k}(k+1) d_{i+j-1 ; s} a_{1}^{i+j} \theta^{i+j+s+k}}{B(i, n-i+1) D(\theta)^{t+j}} .
$$

(3.27) is the order statistics.

To obtain the pdf of smallest order statistics, substituting $i=1$ into (3.27) we get

$$
g_{1 ; n}(x ; p)=\sum_{j=0}^{n-1} \sum_{s=0}^{\infty} \sum_{k=0}^{\infty} w_{1, j, s, k} x^{\beta-1}\{1-(1-G(x))\}^{(j+s+k+1)}, \quad 0<x<\infty
$$

where

$$
w_{1, j, s, k}=\frac{n q(x)\left(\begin{array}{c}
n-1 \\
j
\end{array}\right)(-1)^{j} b_{k}(k+1) d_{j ; s} a_{1}^{j+1} \theta^{j+s+k+1}}{D(\theta)^{j+1}} .
$$

The pdf of largest order statistics is obtained by substituting $i=n$ into (3.27) to get

$$
g_{1 ; n}(x ; p)=\sum_{s+0}^{\infty} \sum_{k+0}^{\infty} w_{n, i, s, k} x^{\beta-1}\{1-(1-G(x))\}^{(j+s+k+n)}, \quad 0<x<\infty
$$

where

$$
w_{n, j, s, k}=\frac{n q(x)(-1)^{j} b_{k}(k+1) d_{n+j-1 ; m} a_{1}^{j+n} \theta^{j+s+k+n}}{D(\theta)^{j+n}} .
$$

\section{Sub-models of the CKWPS Family of Distribution}

In this section, some sub-models of the CKWPS family of distributions are studied. In particular, the Complementary Kumaraswamy Weibull Poisson (CKWP) distribution is discussed in details.

The sub-models considered are as follows:

1. For $D(\theta)=e^{\theta}-1$, the CKWPS family of distribution reduces to the CKWP distribution with cdf 


$$
F_{(x ; a, b, \alpha, \theta)}(x)=\frac{\left[e^{\theta}\left(1-\left(1-\left[1-\exp (-\alpha x)^{\beta}\right]^{a}\right)^{b}\right)\right]-1}{e^{\theta}-1}, \quad x, a, b, \theta, \beta>0 .
$$

2. For $D(\theta)=\theta(1-\theta)^{-1}$, the CKWPS family of distribution reduces to the Complementary Kumaraswamy Weibull Geometric (CKWG) distribution with cdf

$$
F_{(x ; a, b, \alpha, \theta)}(x)=\frac{\theta\left[1-\theta\left(1-\left(1-\left[1-\exp (-\alpha x)^{\beta}\right]^{a}\right)^{b}\right)\right]^{-1}}{\theta[1-\theta]^{-1}}, \quad x, a, b, \theta>0 .
$$

3. For $D(\theta)=(\theta+1)^{m}-1$, the CKWPS family of distribution reduces to the Complementary Kumaraswamy Weibull Binomial (CKWB) distribution with cdf

$$
F_{(x ; a, b, \alpha, \theta)}(x)=\frac{\left[1+\theta\left(1-\left(1-\left[1-\exp (-\alpha x)^{\beta}\right]^{a}\right)^{b}\right)\right]^{m}-1}{(1+\theta)^{m}-1}, \quad x, a, b, \theta>0 .
$$

4. For $D(\theta)=-\operatorname{In}(1-\theta)$, the CKWPS family of distribution reduces to the Complementary Kumaraswamy Weibull Logarithmic (CKWL) distribution with cdf

$$
F_{(x ; a, b, \alpha, \theta)}(x)=\frac{-\operatorname{In}\left\{1-\theta\left[1-\left(1-\left[1-\exp (-\alpha x)^{\beta}\right]^{a}\right)^{b}\right]\right\}}{-\operatorname{In}(1-\theta)}, x, a, b>0,0<\theta<1
$$

5. For $a=b=1$ and $D(\theta)=e^{\theta}-1$, the CKWPS family of distribution reduces to the Complementary Weibull Poisson (CWP) distribution with cdf

$$
F_{(x ; \alpha, \theta)}(x)=\frac{\left[e^{\theta}\left(1-\left(1-\left[1-\exp (-\alpha x)^{\beta}\right]\right)\right)\right]-1}{e^{\theta}-1}, \quad x, a, b, \theta>0 .
$$

6. For $a=b=1$ and $D(\theta)=\theta(1-\theta)^{-1}$, the CKWPS family of distribution reduces to the Complementary Weibull Geometric (CWG) distribution with cdf

$$
F_{(x ; \alpha, \theta)}(x)=\frac{\theta\left[1-\theta\left(1-\left(1-\left[1-\exp (-\alpha x)^{\beta}\right]\right)\right)\right]^{-1}}{\theta[1-\theta]^{-1}}, \quad x, a, b, \theta>0 .
$$


7. For $a=b=1$ and $D(\theta)=(\theta+1)^{m}-1$, the CKWPS family of distribution reduces to the Complementary Weibull Binomial (CWB) distribution with cdf

$$
F_{(x ; \alpha, \theta)}(x)=\frac{\left[1+\theta\left(1-\left(1-\left[1-\exp (-\alpha x)^{\beta}\right]\right)\right)\right]^{m}-1}{(1+\theta)^{m}-1}, \quad x, a, b, \theta>0 .
$$

8. For $a=b=1$ and $D(\theta)=-\operatorname{In}(1-\theta)$, the CKWPS family of distribution reduces to the Complementary Weibull Logarithmic (CWL) distribution with cdf

$$
F_{(x ; \alpha, \theta)}(x)=\frac{-\operatorname{In}\left\{1-\theta\left[1-\left(1-\left[1-\exp (-\alpha x)^{\beta}\right]\right)\right]\right\}}{-\operatorname{In}(1-\theta)}, \quad x, a, b>0, \quad 0<\theta<1 .
$$

9. For $\alpha=1$ and $D(\theta)=e^{\theta}-1$, the CKWPS family of distribution reduces to the Complementary Kumaraswamy Standard Weibull Poisson (CKSWP) distribution with $\operatorname{cdf}$

$$
F_{(x ; a, b, \theta)}(x)=\frac{\left[e^{\theta}\left(1-\left(1-\left[1-\exp (-x)^{\beta}\right]^{a}\right)^{b}\right)\right]-1}{e^{\theta}-1}, \quad x, a, b, \theta>0 .
$$

10. For $\alpha=1$ and $D(\theta)=\theta(1-\theta)^{-1}$, the CKWPS family of distribution reduces to the Complementary Kumaraswamy Standard Weibull Geometric (CKSWG) distribution with cdf

$$
F_{(x ; a, b, \theta)}(x)=\frac{\theta\left[1-\theta\left(1-\left(1-\left[1-\exp (-x)^{\beta}\right]^{a}\right)^{b}\right)\right]^{-1}}{\theta[1-\theta]^{-1}}, \quad x, a, b, \theta>0 .
$$

11. For $\alpha=1$ and $D(\theta)=(\theta+1)^{m}-1$, the CKWPS family of distribution reduces to the Complementary Kumaraswamy Standard Weibull Binomial (CKSWB) distribution with cdf

$$
F_{(x ; a, b, \theta)}(x)=\frac{\left[1+\theta\left(1-\left(1-\left[1-\exp (-x)^{\beta}\right]^{a}\right)^{b}\right)\right]^{m}-1}{(1+\theta)^{m}-1}, \quad x, a, b, \theta>0 .
$$


12. For $\alpha=1$ and $D(\theta)=-\operatorname{In}(1-\theta)$, the CKWPS family of distribution reduces to the Complementary Kumaraswamy Standard Weibull Logarithmic (CKSWL) distribution with cdf

$$
F_{(x ; a, b, \theta)}(x)=\frac{-\operatorname{In}\left\{1-\theta\left[1-\left(1-\left[1-\exp (-x)^{\beta}\right]^{a}\right)^{b}\right]\right\}}{-\operatorname{In}(1-\theta)}, x, a, b>0,0<\theta<1
$$

13. For $\alpha=1, a=b=1$ and $D(\theta)=e^{\theta}-1$, the CKWPS family of distribution reduces to the Complementary Standard Weibull Poisson (CSWP) distribution with cdf

$$
F_{(x ; \theta)}(x)=\frac{\left[e^{\theta}\left(1-\left(1-\left[1-\exp (-x)^{\beta}\right]\right)\right)\right]-1}{e^{\theta}-1}, \quad x, a, b, \theta>0 .
$$

14. For $\alpha=1, a=b=1$ and $D(\theta)=\theta(1-\theta)^{-1}$, the CKWPS family of distribution reduces to the Complementary Standard Weibull Geometric (CSWG) distribution with $\operatorname{cdf}$

$$
F_{(x ; \theta)}(x)=\frac{\theta\left[1-\theta\left(1-\left(1-\left[1-\exp (-x)^{\beta}\right]\right)\right)\right]^{-1}}{\theta[1-\theta]^{-1}}, \quad x, a, b, \theta>0 .
$$

15. For $\alpha=1, \quad a=b=1$ and $D(\theta)=(\theta+1)^{m}-1$, the CKWPS family of distribution reduces to the Complementary Standard Weibull Binomial (CSWB) distribution with cdf

$$
F_{(x ; \theta)}(x)=\frac{\left[1+\theta\left(1-\left(1-\left[1-\exp (-x)^{\beta}\right]\right)\right)\right]^{m}-1}{(1+\theta)^{m}-1}, \quad x, a, b, \theta>0 .
$$

16. For $\alpha=1, a=b=1$ and $D(\theta)=-\operatorname{In}(1-\theta)$, the CKWPS family of distribution reduces to the Complementary Standard Weibull Logarithmic (CSWL) distribution with cdf

$$
F_{(x ; \theta)}(x)=\frac{-\operatorname{In}\left\{1-\theta\left[1-\left(1-\left[1-\exp (-x)^{\beta}\right]\right)\right]\right.}{-\operatorname{In}(1-\theta)}, \quad x, a, b>0, \quad 0<\theta<1 .
$$




\subsection{The complementary Kumaraswamy Weibull Poisson (CKWP) distribution}

Here, the pdf, reliability, hazard function, quantile and moments for the CKWP distribution are studied. The pdf of the CKWP distribution corresponding to (33) is given as

$$
\begin{aligned}
f_{C K W P}(x)= & \frac{a b \alpha \beta \theta x^{\beta-1}}{\mathrm{e}^{\theta}-1} \exp (-\alpha x)^{\beta}\left(1-\exp (-\alpha x)^{\beta}\right)^{a-1}\left(1-\left[1-\exp (-\alpha x)^{\beta}\right]^{a}\right)^{b-1} \\
& \times\left\{\exp \left(\theta\left[1-\left(1-\left[1-\exp (-\alpha x)^{\beta}\right]^{a}\right)^{b}\right]\right)-1\right\}, \quad x, \alpha, a, \beta, b, \theta>0
\end{aligned}
$$

where $a, b, \beta$ are shape parameters and $\alpha$ is a scale parameter.

The plots of the pdf and cdf for the CKWP distribution for some selected parameter values are presented in Figures 1 and 2 respectively.

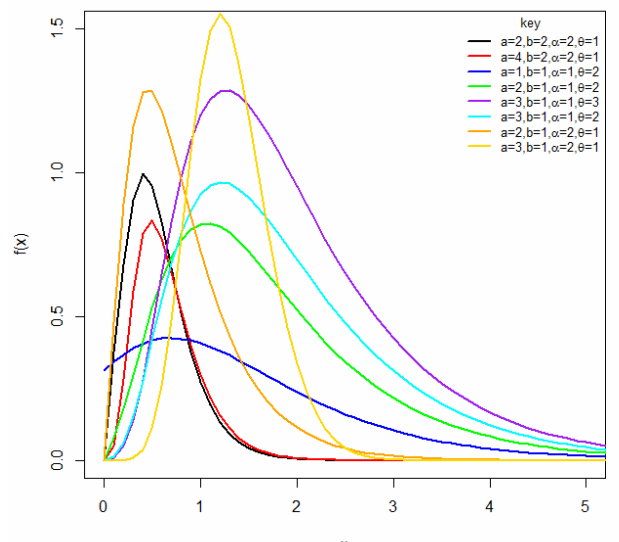

Figure 1. The cdf plot of the CKWP Figure 2. The cdf plot of the CKWP distribution density function for some distribution function for some parameter parameter values.

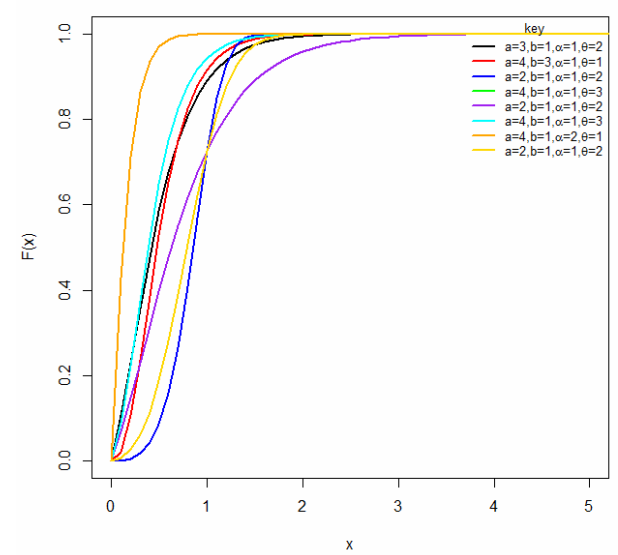
values.

The survival function of the CKWP distribution is given by

$$
S_{C K E P}(x)=\frac{\left(e^{\theta}-1\right)-\exp \left\{\theta\left[1-\left(1-\left[1-\exp \left[\theta \exp \left(-\alpha x^{\beta}\right)\right]\right]^{a}\right)^{b}\right]\right\}-1}{\left(e^{\theta}-1\right)},
$$


The plot of the survival function for the CKWP distribution for some selected parameter values is presented in Figures 3.

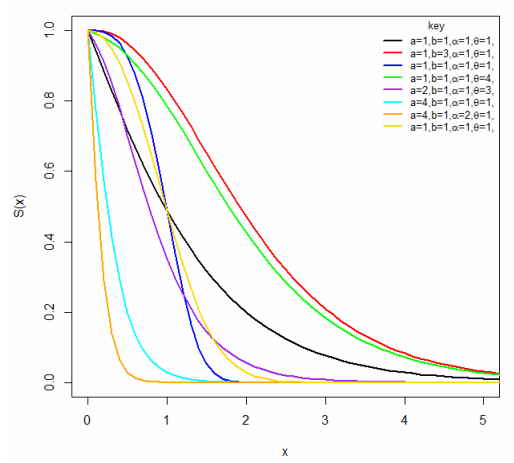

Figure 3. Plot of the CKWP distribution survival function for some parameter values.

The hazard rate function of the CKWP distribution is given by

$$
h_{C K W P}(x)=\frac{a b \alpha \beta \theta x^{\beta-1} \exp (-\alpha x)^{\beta}\left(1-\exp (-\alpha x)^{\beta}\right)^{a-1}\left(1-\left[1-\exp (-\alpha x)^{\beta}\right]^{a}\right)^{b-1}}{\left(e^{\theta}-1\right)-\exp \left\{\theta\left[1-\left(1-\left[1-\exp (-\alpha x)^{\beta}\right]^{a}\right)^{b}\right]\right\}-1}
$$

The plot of the hazard rate function for the CKWP distribution for some selected parameter values is given in Figures 4.

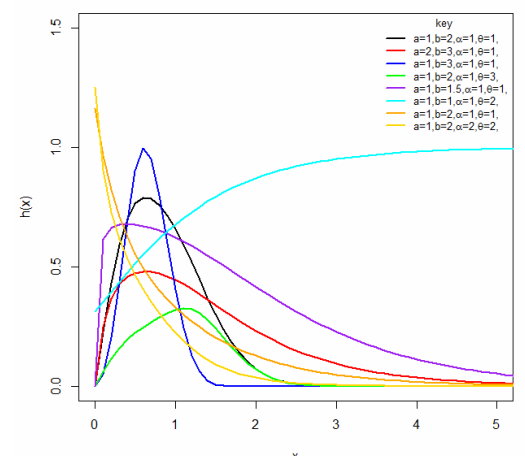

Figure 4. Plot of the CKWP distribution hazard rate functions for some parameter values. 
Figure 4 clearly shows that the hazard rate function exhibits different shapes relative to different values of the parameters.

The quantile function of the CKWP distribution is obtained by substituting $D(\theta)=e^{\theta}-1$ and $D^{-1}(\theta)=e^{\theta}$ into (14) is given by

$$
Q(u)=\frac{1}{\alpha}\left(-\log \left\{1-\left[1-\left(1-\frac{e^{\theta} u\left(e^{\theta}-1\right)}{\theta}\right)^{\frac{1}{b}}\right]^{\frac{1}{a}}\right\}\right.
$$

where $u$ is a uniform random variable on unit interval $(0,1)$. The median $\left(Q_{2}\right)$ of the CKWP distribution is obtained by setting $u=0.5$ in (4.20) to get

$$
Q(u)=\frac{1}{\alpha}\left(-\log \left\{1-\left[1-\left(1-\frac{e^{\theta}\left(e^{\theta}-1\right)}{2 \theta}\right)^{\frac{1}{b}}\right]^{\frac{1}{a}}\right\}\right.
$$

The first moments about the origin (the mean) of the CKWP distribution is obtained by setting $r=1$ in (3.13), to obtain

$$
E(x)=\mu_{r}^{\prime}=\frac{\Gamma(2)}{\alpha\left(e^{\theta}-1\right)} \sum_{m=1}^{\infty} \sum_{j=0}^{m-1} a_{m} \theta^{m} m\left(\begin{array}{c}
m-1 \\
j
\end{array}\right)(-1)^{j} \frac{1}{(j+1)^{2}} .
$$

The variance of the CKWP distribution is given by

$$
\begin{aligned}
\operatorname{Var}(X)= & \sigma^{2}=\mu_{2}^{\prime}-\left(\mu_{1}^{\prime}\right)^{2} \\
= & {\left[\frac{\Gamma(3)}{\alpha\left(e^{\theta}-1\right)} \sum_{m=1}^{\infty} \sum_{j=0}^{m-1} a_{m} \theta^{m} m\left(\begin{array}{c}
m-1 \\
j
\end{array}\right)(-1)^{j} \frac{1}{(j+1)^{3}}\right] } \\
& -\left[\frac{\Gamma(2)}{\alpha\left(e^{\theta}-1\right)} \sum_{m=1}^{\infty} \sum_{j=0}^{m-1} a_{m} \theta^{m} m\left(\begin{array}{c}
m-1 \\
j
\end{array}\right)(-1)^{j} \frac{1}{(j+1)^{2}}\right]^{2} .
\end{aligned}
$$


The moment generating function of the CKWPS distribution is given by

$$
E\left(e^{t X}\right)=M_{x}(t)=\frac{\Gamma(2)}{\alpha\left(e^{\theta}-1\right)} \sum_{r=0}^{\infty} \sum_{m=1}^{\infty} \sum_{j=0}^{m-1} \frac{t^{r}}{r !} a_{m} \theta^{m} m\left(\begin{array}{c}
m-1 \\
j
\end{array}\right)(-1)^{j} \frac{1}{(j+1)^{2}} .
$$

\section{Inferences}

\subsection{Maximum likelihood estimation}

Let $x_{1}, x_{2}, x_{3}, \ldots, x_{n}$ denote a random sample drawn from the CKWP distribution with parameters $\theta, \alpha, \beta, a$ and $b$.

The pdf of the CKWP distribution in (4.19) can be rewritten as

$$
\begin{aligned}
f(x)= & {\left[\frac{a b \alpha \beta \theta}{\left(e^{\theta}-1\right)^{2 a b}}\right] x^{\beta-1} e^{-\alpha x^{\beta}} \exp \left(\theta e^{-\alpha x^{\beta}}\right) u^{a-1}(x)\left[\left(e^{\theta}-1\right)^{a}-u^{a}(x)\right]^{b-1} } \\
& \times t\left\{1-\left[\left(e^{\theta}-1\right)^{a}-u^{a}(x)\right]^{b}\right\},
\end{aligned}
$$

where $u(x)=\exp \left(\theta e^{-\alpha x^{\beta}}\right)-1$.

The likelihood function $f\left(x_{1}, x_{2}, x_{3}, \ldots, x_{n} ; \theta, \alpha, \beta, a, b\right)$ defined to be the joint density of the random variables $x_{1}, x_{2}, x_{3}, \ldots, x_{n}$ is given as

$$
L(x ; \alpha, \beta, a, b, \theta)=\prod_{i=1}^{n} f\left(x_{i} ; \alpha, \beta, a, b, \theta\right) .
$$

The likelihood of the CKWP distribution function is given by

$$
\begin{aligned}
L(x ; \alpha, \beta, a, b, \theta) & =\prod_{i=1}^{n}\left[\frac{a b \alpha \beta \theta}{\left(e^{\theta}-1\right)^{2 a b}}\right] x^{\beta-1} e^{-\alpha x^{\beta}} \exp \left(\theta e^{-\alpha x^{\beta}}\right) u^{a-1}(x) \\
& \times\left[\left(e^{\theta}-1\right)^{a}-u^{a}(x)\right]^{b-1} t\left\{1-\left[\left(e^{\theta}-1\right)^{a}-u^{a}(x)\right]^{b}\right\}
\end{aligned}
$$

and

$$
L=n \log (a)+n \log (b)+n \log (\alpha)+n \log (\beta)+n \log (\theta)-n(2 a b) \log \left(e^{\theta}-1\right)
$$




$$
\begin{aligned}
& +(\beta+1) \sum_{i=1}^{n} \log \left(x_{i}\right)-\alpha \sum_{i=1}^{n} x_{i}^{\beta}+\theta \sum_{i=1}^{n} e^{-\alpha x_{i}^{\beta}}+(a-1) \sum_{i=1}^{n} \log \left[u\left(x_{i}\right)\right] \\
& +(b-1) \sum_{i=1}^{n} \log \left[\left(e^{\theta}-1\right)^{a}-u^{a}\left(x_{i}\right)\right]+t b \sum_{i=1}^{n} \log \left[\left(e^{\theta}-1\right)^{a}-u^{a}\left(x_{i}\right)\right] .
\end{aligned}
$$

Let $\Theta=(\alpha, \beta, a, b, \theta)^{T}$ be the unknown parameter vector. The score vector which is the gradient of the log-likelihood function with respect to the parameters being estimated is given by

$$
U(\Theta)=\left(\frac{\partial L}{\partial a}, \frac{\partial L}{\partial b}, \frac{\partial L}{\partial \alpha}, \frac{\partial L}{\partial \beta}, \frac{\partial L}{\partial \theta}\right)^{T}
$$

The maximum likelihood estimate of $\Theta$ can be obtained by solving the non-linear system of equation $U_{n}(\Theta)=0$. Thus

$$
\begin{aligned}
\frac{\partial L}{\partial a}= & \frac{n}{a}-2 n b \log \left(e^{\theta}-1\right)+\sum_{i=1}^{n} \log \left[u\left(x_{i}\right)\right] \\
& +(b-1) \sum_{i=1}^{n}\left[\frac{\left(e^{\theta}-1\right)^{a} \log \left[\left(e^{\theta}-1\right)^{a}\right]-u^{a}\left(x_{i}\right) \log \left[u\left(x_{i}\right)\right]}{\left(e^{\theta}-1\right)^{a}-\left[u\left(x_{i}\right)\right]^{a}}\right] \\
& +t b \sum_{i=1}^{n}\left[\frac{\left(e^{\theta}-1\right)^{a} \log \left[\left(e^{\theta}-1\right)^{a}\right]-u^{a}\left(x_{i}\right) \log \left[u\left(x_{i}\right)\right]}{\left(e^{\theta}-1\right)^{a}-\left[u\left(x_{i}\right)\right]^{a}}\right]
\end{aligned}
$$

$$
\begin{aligned}
\frac{\partial L}{\partial b}= & \frac{n}{b}-2 n a \log \left(e^{\theta}-1\right)+\sum_{i=1}^{n} \log \left[u\left(x_{i}\right)\right]+\sum_{i=1}^{n}\left[\left(e^{\theta}-1\right)^{a}-u^{a}\left(x_{i}\right)\right] \\
& +t \sum_{i=1}^{n}\left[\left(e^{\theta}-1\right)^{a}-u^{a}\left(x_{i}\right)\right]
\end{aligned}
$$

$$
\begin{aligned}
\frac{\partial L}{\partial \alpha}= & \frac{n}{\alpha}-\sum_{i=1}^{n} x_{i}^{\beta}-\theta \sum_{i=1}^{n} x_{i}^{\beta} e^{-\alpha x_{i}{ }^{\beta}}+(a-1) \sum_{i=1}^{n} \frac{\frac{\partial u\left(x_{i}\right)}{\partial x}}{u\left(x_{i}\right)}-(b-1) \sum_{i=1}^{n} \frac{a\left[u\left(x_{i}\right)\right]^{\alpha-1} \frac{\partial u\left(x_{i}\right)}{\partial x}}{\left(e^{\theta}-1\right)^{a}-\left[u\left(x_{i}\right)\right]^{a}} \\
& +t b \sum_{i=1}^{n} \frac{a\left[u\left(x_{i}\right)\right]^{\alpha-1} \frac{\partial u\left(x_{i}\right)}{\partial x}}{\left(e^{\theta}-1\right)^{a}-\left[u\left(x_{i}\right)\right]^{a}}
\end{aligned}
$$




$$
\begin{aligned}
\frac{\partial L}{\partial \beta}= & \frac{n}{\beta}-\sum_{i=1}^{n} \log \left(x_{i}\right)-\alpha \sum_{i=1}^{n} x_{i}^{\beta} \log \left(x_{i}\right)-\alpha \theta \sum_{i=1}^{n} x_{i}^{\beta} \log \left(x_{i}\right) e^{-\alpha x_{i}{ }^{\beta}}+(a-1) \sum_{i=1}^{n} \frac{\frac{\partial u\left(x_{i}\right)}{\partial x}}{u\left(x_{i}\right)} \\
& -(b-1) \sum_{i=1}^{n} \frac{a\left[u\left(x_{i}\right)\right]^{\alpha-1} \frac{\partial u\left(x_{i}\right)}{\partial x}}{\left(e^{\theta}-1\right)^{a}-\left[u\left(x_{i}\right)\right]^{a}}+t b \sum_{i=1}^{n} \frac{\left.a\left[u\left(x_{i}\right)\right]^{\alpha-1} \frac{\partial u\left(x_{i}\right)}{\partial x}-1\right)^{a}-\left[u\left(x_{i}\right)\right]^{a}}{\partial \theta} \\
\frac{\partial L}{\theta}= & \frac{n a b e^{\theta}}{e^{\theta}-1}+\sum_{i=1}^{n} e^{-\alpha x_{i}^{\beta}}+(a-1) \sum_{i=1}^{n} \frac{\frac{\partial u\left(x_{i}\right)}{\partial \theta}}{u\left(x_{i}\right)} \\
& +a(b-1) \sum_{i=1}^{n} \frac{e^{\theta}\left(e^{\theta}-1\right)^{a-1}-\left[u\left(x_{i}\right)\right]^{\alpha-1} \frac{\partial u\left(x_{i}\right)}{\partial \theta}}{\left(e^{\theta}-1\right)^{a}-\left[u\left(x_{i}\right)\right]^{a}} \\
& +t b a \sum_{i=1}^{n} \frac{e^{\theta}\left(e^{\theta}-1\right)^{a-1}-\left[u\left(x_{i}\right)\right]^{\alpha-1} \frac{\partial u\left(x_{i}\right)}{\partial \theta}}{\left(e^{\theta}-1\right)^{a}-\left[u\left(x_{i}\right)\right]^{a}}
\end{aligned}
$$

Setting (5.6), (5.7), (5.8), (5.9) and (5.10) to zero and solving for the solution of the non-linear system of equations produce the maximum likelihood estimates of parameters $\hat{a}, \hat{b}, \hat{\alpha}, \hat{\beta}$ and $\hat{\theta}$. However these solutions can only be obtained numerically with the aid of suitable statistical software like R, SAS etc. Hence, some datasets are considered in the next section to fit the proposed distribution with other related distributions using "maxLik" package in R software.

\section{Applications}

This section presents a real life datasets, the descriptive statistics, graphical summary and application. Four models (Kumaraswamy-Weibull Distribution (KWD), Kumaraswamy-Modified Weibull Distribution (KMWD), Kumaraswamy Exponential Weibull Distribution (KEWD) and Kumaraswamy-Weibull Poisson Distribution (KWPD)) are applied to a real life dataset alongside the proposed (Complementary Kumaraswamy-Weibull-Poisson Distribution (CKWPD)). The performance of these distributions are evaluated and compared using some Model Selection Information Criteria (MSIC) which include AIC (Akaike Information Criterion), CAIC (Consistent Akaike Information Criterion), BIC (Bayesian Information Criterion) and HQIC (Hannan Quin Information Criterion). The MSIC are given as follows: 


$$
\begin{aligned}
& A I C=-2 l l+2 k, \quad B I C=-2 l l+k \log (n), \quad C A I C=-2 l l+\frac{2 k n}{(n-k-1)} \text { and } \\
& H Q I C=-2 l l+2 k \log [\log (n)],
\end{aligned}
$$

where $l l$ denotes the log-likelihood value evaluated with the maximum likelihood estimates, $k$ is the number of model parameters and $n$ is the sample size. The model with the lowest values of these statistics would be chosen as the best model to fit the dataset.

Data: The data set consists of 63 observations of the strengths of $1.5 \mathrm{~cm}$ glass fibers, originally obtained by workers at the UK National Physical Laboratory. Unfortunately, the units of measurement are not given in the paper. It has been used by [16], [17], [18], [19], [20], [21], [22], [23]. It is given as

$0.55,1.28,1.51,1.61,1.70,2.00,0.74,1.29,1.52,1.62,1.7,2.01,0.77,1.3,1.53,1.62$, $1.73,2.24,0.81,1.36,1.54,1.63,1.76,0.84,1.39,1.55,1.64,1.76,0.93,1.42,1.55,1.66$, $1.77,1.04,1.48,1.58,1.66,1.78,1.11,1.48,1.59,1.66,1.81,1.13,1.49,1.6,1.67,1.82$, $1.24,1.49,1.61,1.68,1.84,1.25,1.5,1.61,1.68,1.84,1.27,1.50,1.61,1.69,1.89$.

Source: Smith and Naylor (1987).

Table 2. Descriptive Statistics for the dataset.

\begin{tabular}{|c|c|c|c|c|c|c|c|c|c|}
\hline No & Minimum & $Q_{1}$ & Median & $Q_{3}$ & Mean & Maximum & Variance & Skewness & Kurtosis \\
\hline 63 & 0.550 & 1.375 & 1.590 & 1.685 & 1.507 & 2.240 & 0.105 & -0.8786 & 3.9238 \\
\hline
\end{tabular}
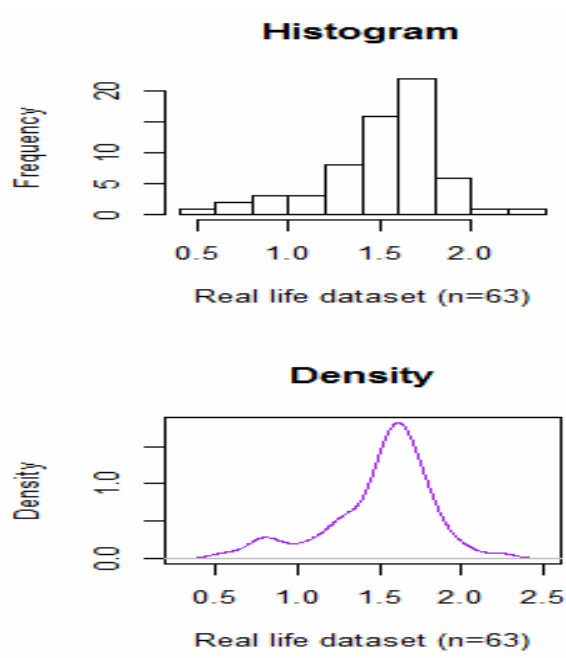
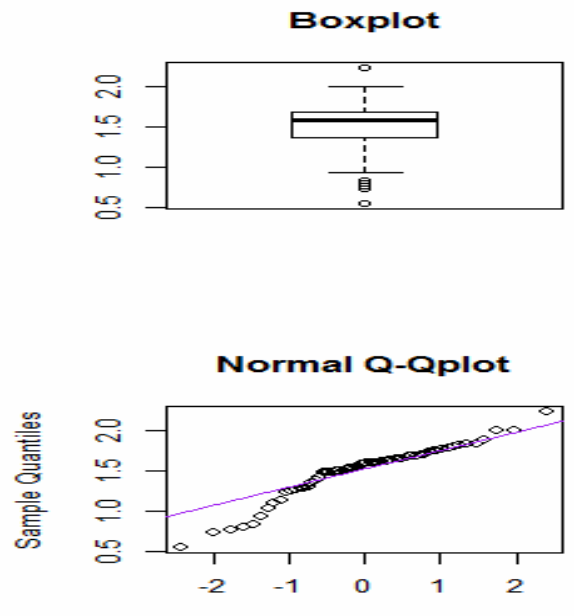

Theoretical Quantiles

Figure 5. A graphical summary for the real life data. 
The result in Table 2 and the graphical display in Figure 5 reveal that the dataset is negatively skewed, and therefore would be flexible for skewed distributions.

Table 3. Performance evaluation of the distributions based on the dataset.

\begin{tabular}{|c|c|c|c|c|c|c|c|}
\hline Distributions & $\begin{array}{l}\text { Parameter } \\
\text { estimates }\end{array}$ & $\begin{array}{l}-l l=(-\log - \\
\text { likelihood } \\
\text { value })\end{array}$ & $A I C$ & CAIC & $B I C$ & $H Q I C$ & Ranks \\
\hline CKWPD & $\begin{array}{l}\hat{\alpha}=0.30530595 \\
\hat{\beta}=0.32521237 \\
\hat{\theta}=0.63305191 \\
\hat{a}=0.14508335 \\
\hat{b}=0.05181175\end{array}$ & 5.393076 & 20.78615 & 21.27796 & 35.0463 & 26.58012 & $1^{\mathrm{st}}$ \\
\hline KWPD & $\begin{array}{l}\hat{\alpha}=0.06819696 \\
\hat{\beta}=0.03372159 \\
\hat{\theta}=0.05090372 \\
\hat{a}=0.01741473 \\
\hat{b}=0.05126014\end{array}$ & 5.581409 & 21.16282 & 21.65462 & 35.4230 & 26.95679 & $2^{\text {nd }}$ \\
\hline KMWD & $\begin{array}{l}\hat{\alpha}=0.07465782 \\
\hat{\beta}=2.42877590 \\
\hat{\theta}=0.001600541 \\
\hat{a}=4.80395492 \\
\hat{b}=5.95925826\end{array}$ & 7.123126 & 24.24625 & 24.73806 & 38.5064 & 30.04022 & $3^{\text {rd }}$ \\
\hline KWD & $\begin{array}{l}\hat{\alpha}=1.34165211 \\
\hat{\beta}=0.00722436 \\
\hat{a}=1.63128742 \\
\hat{b}=0.01331726\end{array}$ & 8.545615 & 25.09123 & 25.41643 & 36.4994 & 29.72641 & $4^{\text {th }}$ \\
\hline KEWD & $\begin{array}{l}\hat{\alpha}=0.3083907 \\
\hat{\beta}=0.5765746 \\
\hat{\theta}=0.1949686 \\
\hat{a}=0.2592218 \\
\hat{b}=0.4121287\end{array}$ & 16.92949 & 43.85899 & 44.35079 & 58.1191 & 49.65296 & $5^{\text {th }}$ \\
\hline
\end{tabular}


Figure 6 displayed the histogram and estimated densities and cdfs of the fitted models for dataset.
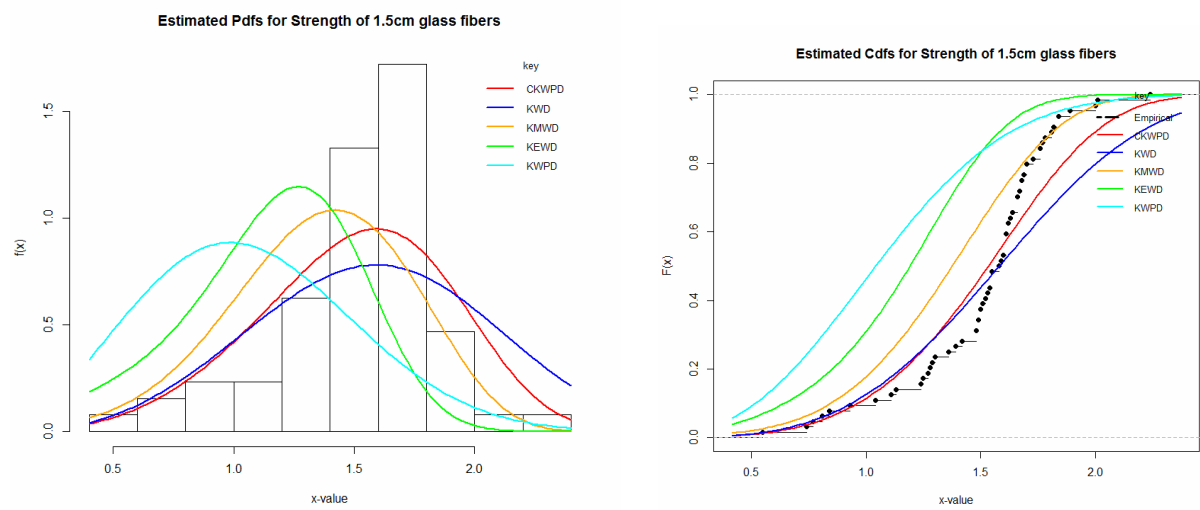

Figure 6. Histogram and plots of the estimated densities (pdfs) and cdfs of the distribution.
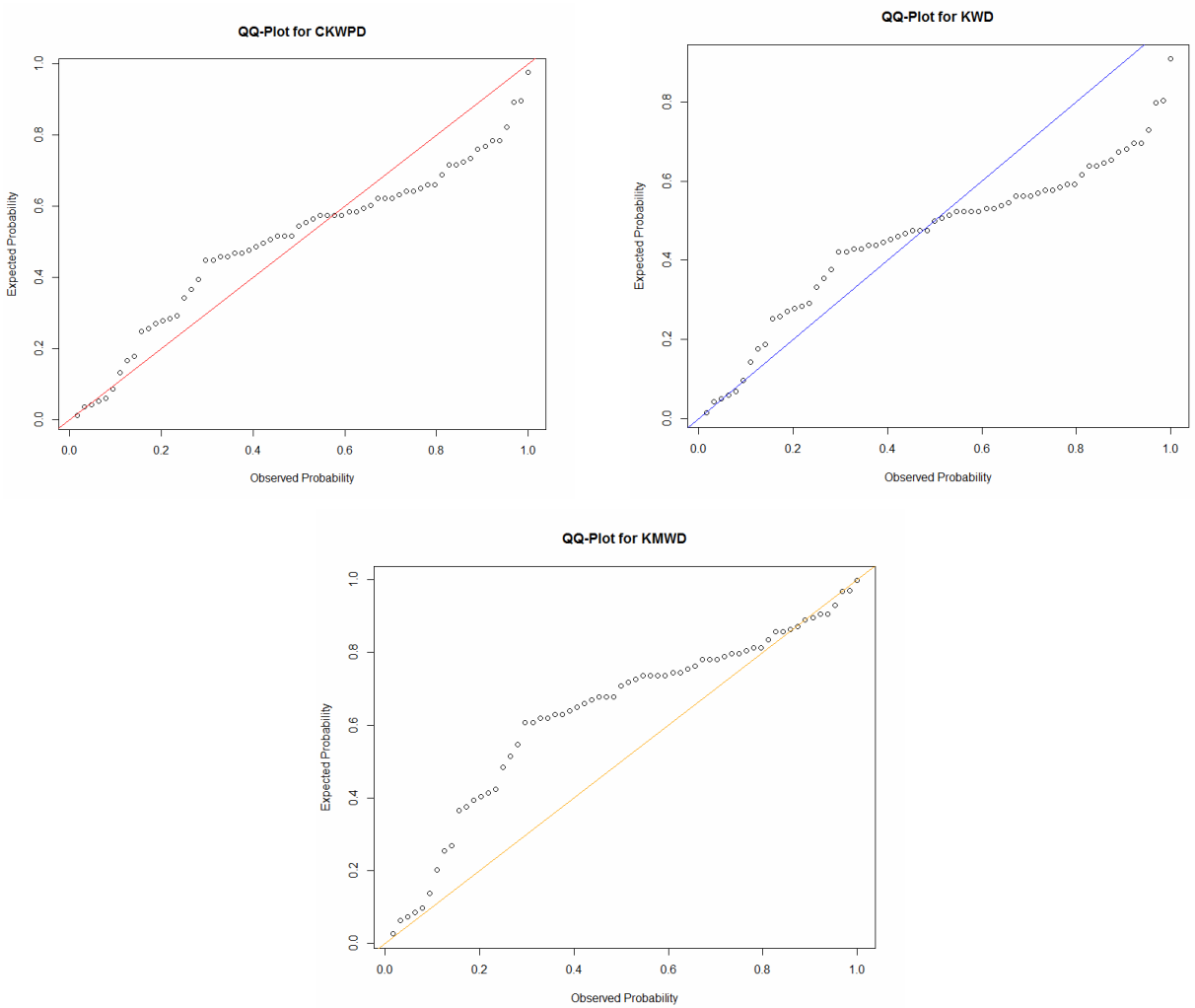

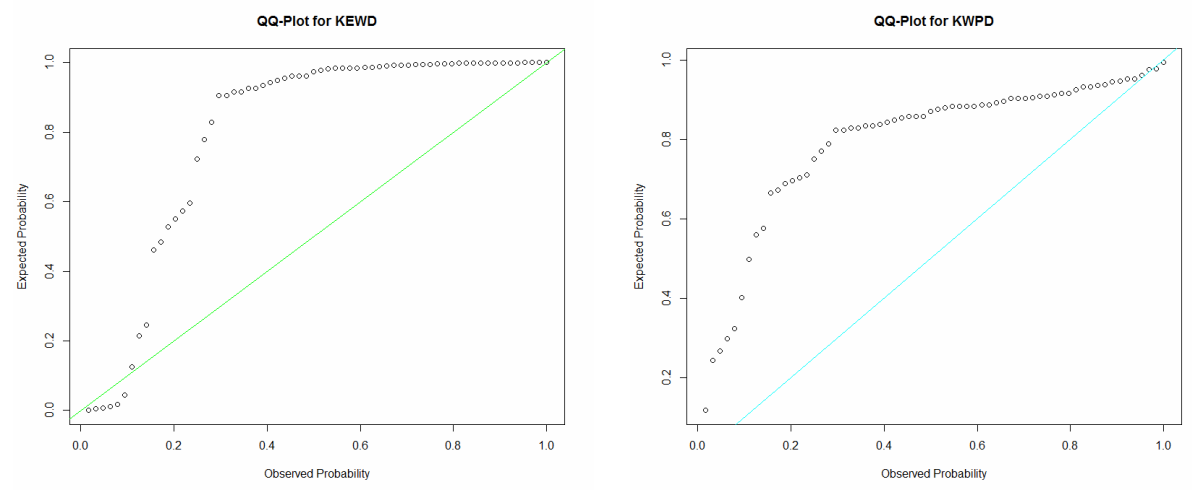

Figure 7. Probability plots for the fit of the distributions based on the dataset.

Table 3 clearly shows that the CKWPD has smallest values of -ll, AIC, BIC, CAIC and HQIC compared to the other four distributions using the real life dataset. This provides evidence to show that the CKWPD fits the real life data better than the other four models. The plot in Figure 6 also reveals that the CKWPD performs better than the KWPD, KMWD, KWD and KEWD in fitting the dataset. Similarly, the probability plots displayed in Figure 7 further provide evidence that the proposed distribution (CKWPD) is more flexible for the dataset than the other four distributions (KWPD, KMWD, KWD and KEWD).

\section{Conclusion}

In this paper we have proposed a new member of the Complementary Kumaraswamy - G Power Series family of distributions obtained by the method of compounding. The properties of the proposed distribution have been studied. In particular a member of this family of distribution called the Complementary Kumaraswamy Weibull Poisson Distribution (CKWPD) or simply CKWP has been extensively discussed. The usefulness of the CKWPD has been investigated by application to a real life dataset. Results from the application show that the CKWPD performs better than the KWD, KMWD, KEWD and KWPD in fitting the dataset.

\section{References}

[1] N. Eugene, C. Lee and F. Famoye, Beta-normal distribution and its applications, Comm. Statist. Theory Methods 31(4) (2002), 497-512. https://doi.org/10.1081/STA-120003130 
[2] K. Adamidis and S. Loukas, A lifetime distribution with decreasing failure rate, Statist. Probab. Lett. 39 (1998), 35-42. https://doi.org/10.1016/S0167-7152(98)00012-1

[3] S. Rezaei and R. Tahmasbi, A new lifetime distribution with increasing failure rate: exponential truncated Poisson, J. Basic Appl. Sci. Res. 2(2) (2012), 1749-1762.

[4] A. S. Hassan, S. M. Assar and K. A. Ali, The complementary Poisson-Lindley class of distributions, International Journal of Advanced Statistics and Probability 3(2) (2015), 146-160. https://doi.org/10.14419/ijasp.v3i2.4624

[5] A. L. Morais and W. Barreto-Souza, A compound class of Weibull and power series distributions, Comput. Statist. Data Anal. 55 (2011), 1410-1425.

https://doi.org/10.1016/j.csda.2010.09.030

[6] E. P. Mahmoudi and A. A. Jafari, Generalized exponential-power series distributions, Comput. Statist. Data Anal. 55 (2012), 4047-4066.

https://doi.org/10.1016/j.csda.2012.04.009

[7] R. B. Silva, M. Bourguignon, C. R. B. Dias and G. M. Cordeiro, The compound class of extended Weibull power series distributions, Comput. Statist. Data Anal. 58 (2013), 352367. https://doi.org/10.1016/j.csda.2012.09.009

[8] R. B. Silva and G. M. Cordeiro, The Burr XII power series distributions: A new compounding family, Braz. J. Probab. Stat. 29(3) (2015), 565-589.

https://doi.org/10.1214/13-BJPS234

[9] G. W. Liyanage and M. Pararai, The Lindley power series class of distributions: model, properties and applications, Journal of Computations and Modeling 5(3) (2015), 35-80.

[10] G. M. Cordeiro and M. de Castro, A new family of generalized distributions, J. Stat. Comput. Simul. 81(7) (2011), 883-898. https://doi.org/10.1080/00949650903530745

[11] A. W. Marshall and I. Olkin, A new method for adding a parameter to a family of distributions with application to the exponential and Weibull families, Biometrika 84 (1997), 641-652. https://doi.org/10.1093/biomet/84.3.641

[12] A. Noack, A class of random variables with discrete distributions, Ann. Math. Statist. 21 (1950), 127-132. https://doi.org/10.1214/aoms/1177729894

[13] O. D. Kosambi, Characteristic properties of series distributions, Proc. Nat. Inst. Sci. India 15 (1949), 109-113.

[14] G. P. Patil, Contribution to the estimation in a class of discrete distributions, Ph.D. Thesis, Ann Arbor, MI: University of Michigan, 1961. 
[15] G. P. Patil, Certain properties of the generalized power series distribution II, Ann. Inst. Statist. Math. 14 (1962), 179-182. https://doi.org/10.1007/BF02868639

[16] R. L. Smith and J. C. Naylor, A comparison of maximum likelihood and Bayesian estimators for the three-parameter Weibull distribution, Appl. Statist. 36 (1987), 358-369. https://doi.org/10.2307/2347795

[17] W. Barreto-Souza, A. I. de Morais and G. M. Cordeiro, The Weibull-geometric distributions, J. Stat. Comput. Simul. 81 (2011), 645-657.

https://doi.org/10.1080/00949650903436554

[18] M. Bourguignon, R. B. Silva and G. M. Cordero, The Weibull-G family of probability distributions, J. Data Sci. 12 (2014), 53-68.

[19] P. E. Oguntunde, O. S. Balogun, H. I. Okagbue and S. A. Bishop, The Weibull exponential distribution: Its properties and applications, J. Appl. Sci. 15(11) (2015), 1305-1311. https://doi.org/10.3923/jas.2015.1305.1311

[20] M. Mansour, G. Aryal, A. Z. Afify and M. Ahmad, The Kumaraswamy exponentiated Fréchet distribution, Pakistan Journal of Statistics 34(3) (2018), 177-193.

[21] T. G. Ieren and A. Yahaya, The Weimal distribution: its properties and applications, Journal of the Nigerian Association of Mathematical Physics 39 (2017), 135-148.

[22] A. Yahaya and T. G. Ieren, On transmuted Weibull-exponential distribution: its properties and applications, Nigerian Journal of Scientific Research 16(3) (2017b), 289-297.

[23] T. G. Ieren, S. O. Oyamakin, A. Yahaya, A. U. Chukwu, A. A. Umar and S. Kuje, On making an informed choice between two Lomax-based continuous probability distributions using lifetime data, Asian J. Prob. Stat. 2(2) (2018), 1-11. https://doi.org/10.9734/ajpas/2018/v2i228780 


\section{Appendix A}

The R functions for cdf, pdf, survival and hazard functions diagrams.

$a=3$

$b=3$

$c=3$

$\mathrm{d}=3$

$t=2$

$\mathrm{u}=\operatorname{runif}(25,0,1)$

$\mathrm{x}=\left(-(1 / \mathrm{c}) * \log \left(1-\left(1-\left(1-(1 / \mathrm{t}) * \log \left(\left(1+\mathrm{u}^{*}(\exp (\mathrm{t})-1)\right)\right)\right)^{\wedge}(1 / \mathrm{b})\right)^{\wedge}(1 / \mathrm{a})\right)\right)^{\wedge}(1 / \mathrm{d})$

pdfckwpd=function $(\mathrm{x}, \mathrm{a}, \mathrm{b}, \mathrm{c}, \mathrm{d}, \mathrm{t})\left(\mathrm{a} * \mathrm{~b}^{*} \mathrm{c}^{*} \mathrm{~d}^{*} \mathrm{t}^{*} \mathrm{x}^{\wedge}(\mathrm{d}-1) * \exp \left(-\mathrm{c}^{*} \mathrm{x}^{\wedge} \mathrm{d}\right) *\left(1-\exp \left(-\mathrm{c} * \mathrm{x}^{\wedge} \mathrm{d}\right)\right)^{\wedge}(\mathrm{a}-1) *(1-(1-\exp (-\right.$ $\left.\left.\left.\left.\mathrm{c}^{*} \mathrm{x}^{\wedge} \mathrm{d}\right)\right)\right)^{\wedge}(\mathrm{b}-1)^{*} \exp \left(\mathrm{t}^{*}\left(1-\left(1-\left(1-\exp \left(-\mathrm{c}^{*} \mathrm{x}^{\wedge} \mathrm{d}\right)^{\wedge} \mathrm{a}\right)^{\wedge} \mathrm{b}\right)\right)\right) /(\exp (\mathrm{t})-1)\right)$

cdfckwpd=function $(x, a, b, c, d, t)\left(\left(\exp \left(t^{*}\left(1-\left(1-\left(1-\exp \left(-c^{*} x^{\wedge} d\right)^{\wedge} a\right)^{\wedge} b\right)\right)\right)-1\right) /(\exp (t)-1)\right)$

sfckwpd=function $(x, a, b, c, d, t)\left(1-\left(\left(\exp \left(t^{*}\left(1-\left(1-\left(1-\exp \left(-c^{*} x^{\wedge} d\right)^{\wedge} a\right)^{\wedge} b\right)\right)\right)-1\right) /(\exp (t)-1)\right)\right)$

hfckwpd=function $(\mathrm{x}, \mathrm{a}, \mathrm{b}, \mathrm{c}, \mathrm{d}, \mathrm{t})\left(\mathrm{a} * \mathrm{~b}^{*} \mathrm{c} * \mathrm{~d}^{*} \mathrm{t}^{*} \mathrm{x}^{\wedge}(\mathrm{d}-1) * \exp \left(-\mathrm{c} * \mathrm{x}^{\wedge} \mathrm{d}\right) *\left(1-\exp \left(-\mathrm{c} * \mathrm{x}^{\wedge} \mathrm{d}\right)\right)^{\wedge}(\mathrm{a}-1) *(1-(1-\exp (-\right.$

$\left.\left.\left.\mathrm{c}^{*} \mathrm{x}^{\wedge} \mathrm{d}\right)\right)\right)^{\wedge}(\mathrm{b}-1)^{*} \exp \left(\mathrm{t}^{*}\left(1-\left(1-\left(1-\exp \left(-\mathrm{c}^{*} \mathrm{x}^{\wedge} \mathrm{d}\right)^{\wedge} \mathrm{a}\right)^{\wedge} \mathrm{b}\right)\right)\right) /(\exp (\mathrm{t})-1) /\left(1-\left(\left(\exp \left(\mathrm{t}^{*}\left(1-\left(1-\left(1-\exp \left(-\mathrm{c}^{*} \mathrm{x}^{\wedge} \mathrm{d}\right)^{\wedge} \mathrm{a}\right)^{\wedge} \mathrm{b}\right)\right)\right)-1\right)\right.\right.$

$/(\exp (\mathrm{t})-1))))$

curve(pdfckwpd(x,1,1,1,1,1),type="n", xlim=c(0,5),ylim=c(0,1.5),ylab="f(x)", main=" ")

curve $(\operatorname{pdfckwpd}(\mathrm{x}, 1,1,1,1,1)$, from=0,to=10,add=T,lwd=2,col="black",lty=1)

curve(pdfckwpd(x,1,3,1,1,1),from=0,to=10, add=T,lwd=2,col="red",lty=1)

curve(pdfckwpd(x,1,1,1,3,1),from=0,to=10,add=T,lwd=2,col="blue",lty=1)

curve $(\operatorname{pdfckwpd}(\mathrm{x}, 1,1,1,1,4)$, from=0,to=10,add=T,lwd=2,col="green",lty=1)

curve $(\operatorname{pdfckwpd}(\mathrm{x}, 2,1,1,1,3)$, from=0,to=10, $\mathrm{add}=\mathrm{T}, 1 \mathrm{wd}=2, \mathrm{col}=$ "purple",lty=1)

curve(pdfckwpd $(x, 4,1,1,1,1)$,from=0,to=10,add=T,lwd=2,col="cyan",lty=1)

curve $(\operatorname{pdfckwpd}(\mathrm{x}, 4,1,2,1,1)$, from=0,to=10, $\mathrm{add}=\mathrm{T}, \mathrm{lwd}=2, \mathrm{col}=$ "orange",lty=1)

curve(pdfckwpd(x,1,1,1,2,1),from=0,to=10,add=T,lwd=2,col="gold",lty=1)

legend(locator(1) ,cex=0.8,title="key",c(expression(paste(a,"=1,",b,"=1,", alpha,"=1,",

beta,"=1,", $\quad$ theta,"=1")), $\quad$ expression(paste(a,"=1,",b,"=3,", $\quad$ alpha,"=1,", $\quad$ beta,"=1,", $\quad$ theta,"=1")), expression(paste(a,"=1,",b,"=1,", alpha,"=1,", beta,"=3,", theta,"=1")), expression(paste(a,"=1,",b,"=1,", alpha,"=1,", beta,"=1,", theta,"=4")), expression(paste(a,"=2,",b,"=1,", alpha,"=1,", beta,"=1,", theta,"=3")), expression(paste(a,"=4,",b,"=1,", alpha,"=1,", beta,"=1,", theta,"=1")), expression(paste(a,"=4,",b,"=1,", alpha,"=2,", beta,"=1,", theta,"=1")), 
expression(paste(a,"=1,",b,"=1,", alpha,"=1,", beta,"=2,",

theta,"=1"))),horiz=FALSE, 1 ty=c(1,1,1,1,1,1,1,1), $1 w d=c(2,2,2,2,2,2,2,2)$,

bty="n",col=c("black","red","blue","green","purple","cyan","orange","gold"))

curve (cdfckwpd(x,1,1,1,1,1),type="n", xlim=c(0,5),ylim=c(0,1),ylab="F(x)", main=" ")

curve $(\operatorname{cdfckwpd}(\mathrm{x}, 1,1,1,1,1)$, from=0,to=10, add=T,lwd=2,col="black",lty=1)

curve $(\operatorname{cdfckwpd}(\mathrm{x}, 1,3,1,1,1)$, from=0,to=10, add=T,lwd=2, col="red",lty=1)

curve $(\operatorname{cdfckwpd}(\mathrm{x}, 1,1,1,3,1)$, from=0,to=10,add=T,lwd=2,col="blue",lty=1)

curve $(\operatorname{cdfckwpd}(\mathrm{x}, 1,1,1,1,4)$, from=0,to=10, add=T,lwd=2,col="green",lty=1)

curve (cdfckwpd(x,2,1,1,1,3),from=0,to=10, add=T,lwd=2,col="purple",lty=1)

curve $(\operatorname{cdfckwpd}(\mathrm{x}, 4,1,1,1,1)$, from=0,to=10, add=T,lwd=2,col="cyan",lty=1)

curve $(\operatorname{cdfckwpd}(\mathrm{x}, 4,1,2,1,1)$, from=0,to=10, add=T,lwd=2,col="orange",lty=1)

curve $(\operatorname{cdfckwpd}(\mathrm{x}, 1,1,1,2,1)$, from=0,to=10, add=T,lwd=2,col="gold",lty=1)

legend(locator(1) ,cex=0.8,title="key",c(expression(paste(a,"=1,",b,"=1,", alpha,"=1,",

beta,"=1,", $\quad$ theta,"=1")), $\quad$ expression(paste(a,"=1,",b,"=3,", alpha,"=1,", $\quad$ beta,"=1,", $\quad$ theta,"=1")), expression(paste(a,"=1,",b,"=1,", alpha,"=1,", beta,"=3,", theta,"=1")),

expression(paste(a,"=1,",b,"=1,", alpha,"=1,", beta,"=1,", theta,"=4")), expression(paste(a,"=2,",b,"=1,", alpha,"=1,", beta,"=1,", theta,"=3")), expression(paste(a,"=4,",b,"=1,", alpha,"=1,", beta,"=1,", theta,"=1")), expression(paste(a,"=4,",b,"=1,", alpha,"=2,", beta,"=1,", theta,"=1")), expression(paste(a,"=1,",b,"=1,", alpha,"=1,", beta,"=2,", theta,"=1"))),horiz=FALSE, 1 ty=c(1,1,1,1,1,1,1,1), $1 \mathrm{wd}=\mathrm{c}(2,2,2,2,2,2,2,2)$, bty="n",col=c("black","red","blue","green","purple","cyan","orange","gold")) curve $($ sfckwpd(x,1,1,1,1,1),type="n", $x \lim =c(0,5), y \lim =c(0,1), y l a b=" S(x) "$, main=" ") curve $(\operatorname{sfckwpd}(\mathrm{x}, 1,1,1,1,1)$, from=0,to=10,add=T,lwd=2,col="black",lty=1) curve $(\operatorname{sfckwpd}(\mathrm{x}, 1,3,1,1,1)$, from=0,to=10, add=T,lwd=2,col="red",lty=1) curve $(\operatorname{sfckwpd}(\mathrm{x}, 1,1,1,3,1)$,from=0,to=10,add=T,lwd=2,col="blue",lty=1) curve $(\operatorname{sfckwpd}(\mathrm{x}, 1,1,1,1,4)$, from=0,to=10,add=T,lwd=2,col="green",lty=1) curve $(\operatorname{sfckwpd}(\mathrm{x}, 2,1,1,1,3)$, from=0,to=10, add=T,lwd=2, col="purple",lty=1) curve $(\operatorname{sfckwpd}(\mathrm{x}, 4,1,1,1,1)$, from=0,to=10, add=T,lwd=2, col="cyan",lty=1) curve $(\operatorname{sfckwpd}(\mathrm{x}, 4,1,2,1,1)$, from=0,to=10, add=T,lwd=2, col="orange",lty=1) curve $(\operatorname{sfckwpd}(\mathrm{x}, 1,1,1,2,1)$, from=0,to=10, add=T,lwd=2, col="gold",lty=1) legend(locator(1) ,cex=0.8,title="key",c(expression(paste(a,"=1,",b,"=1,", alpha,"=1,", beta,"=1,", theta,"=1,")), expression(paste(a,"=1,",b,"=3,", alpha,"=1,", beta,"=1,", theta,"=1,")), 
expression(paste(a,"=1,",b,"=1,", alpha,"=1,", beta,"=3,", theta,"=1,")), expression(paste(a,"=1,",b,"=1,", alpha,"=1,", beta,"=1,", theta,"=4,")), expression(paste(a,"=2,",b,"=1,", alpha,"=1,", beta,"=1,", theta,"=3,")), expression(paste(a,"=4,",b,"=1,", alpha,"=1,", beta,"=1,", theta,"=1,")), expression(paste(a,"=4,",b,"=1,", alpha,"=2,", beta,"=1,", theta,"=1,")), expression(paste(a,"=1,",b,"=1,", alpha,"=1,", beta,"=2,",

theta,"=1,"))), horiz=FALSE,lty=c(1,1,1,1,1,1,1,1),lwd=c(2,2,2,2,2,2,2,2), bty="n",col=c("black","red","blue","green","purple","cyan","orange","gold"))

curve(hfckwpd(x,1,1,1,1,1),type="n", xlim=c(0,5),ylim=c(0,1.5),ylab="h(x)", main=" ")

curve $($ hfckwpd $(x, 1,1,1,1,1)$, from=0,to=10,add=T,lwd=2,col="black",lty=1)

curve(hfckwpd(x,1,3,1,1,1), from=0,to=10, add=T,lwd=2,col="red",lty=1)

curve(hfckwpd(x,1,1,1,3,1),from=0,to=10, add=T,lwd=2,col="blue",lty=1)

curve(hfckwpd(x,1,1,1,1,4),from=0,to=10,add=T,lwd=2,col="green",lty=1)

curve(hfckwpd(x,2,1,1,1,3),from=0,to=10,add=T,lwd=2,col="purple",lty=1)

curve(hfckwpd(x,4,1,1,1,1),from=0,to=10, add=T,lwd=2,col="cyan",lty=1)

curve(hfckwpd(x,4,1,2,1,1),from=0,to=10,add=T,lwd=2,col="orange",lty=1)

curve(hfckwpd(x,1,1,1,2,1),from=0,to=10, add=T,lwd=2,col="gold",lty=1)

legend(locator(1) ,cex=0.8,title="key",c(expression(paste(a,"=1,",b,"=1,", alpha,"=1,", beta,"=1,", theta,"=1,")), expression(paste(a,"=1,",b,"=3,", alpha,"=1,", beta,"=1,", theta,"=1,")), expression(paste(a,"=1,",b,"=1,", alpha,"=1,", beta,"=3,", theta,"=1,")), expression(paste(a,"=1,",b,"=1,", alpha,"=1,", beta,"=1,", theta,"=4,")), expression(paste(a,"=2,",b,"=1,", alpha,"=1,", beta,"=1,", theta,"=3,")), expression(paste(a,"=4,",b,"=1,", alpha,"=1,", beta,"=1,", theta,"=1,")), expression(paste(a,"=4,",b,"=1,", alpha,"=2,", beta,"=1,", theta,"=1,")), expression(paste(a,"=1,",b,"=1,", alpha,"=1,", beta,"=2,",

theta,"=1,"))), horiz=FALSE,lty=c(1,1,1,1,1,1,1,1),lwd=c(2,2,2,2,2,2,2,2),

bty="n",col=c("black","red","blue","green","purple","cyan","orange","gold"))

\section{Appendix B}

The R function estimates the model parameters.

library(AdequacyModel)

$\mathrm{x}=\mathrm{c}(0.55,1.28,1.51,1.61,1.70,2.00,0.74,1.29,1.52,1.62,1.7,2.01,0.77,1.3,1.53,1.62,1.73,2.24,0.81,1.36,1.54,1$

$.63,1.76,0.84,1.39,1.55,1.55,1.64,1.76,0.93,1.42,1.55,1.66,1.77,1.04,1.48,1.58,1.66,1.78,1.11,1.48,1.59,1.66$

,1.81,1.13,1.49,1.6,1.67,1.82,1.24,1.49,1.61,1.68,1.84,1.25,1.5,1.61,1.68,1.84,1.27,1.50,1.61,1.69,1.89)

pdfckwpd=function $(\mathrm{x}$, par $)\{$ 
alpha=par[1]

beta=par[2]

theta=par[3]

$\mathrm{a}=\operatorname{par}[4]$

$\mathrm{b}=\operatorname{par}[5]$

$a^{*} b^{*}$ alpha*beta*theta* $x^{\wedge}\left(\text { beta-1)*exp(-alpha* } x^{\wedge} \text { beta }\right)^{*}\left(1-\exp \left(- \text { alpha* } x^{\wedge} \text { beta }\right)\right)^{\wedge}(a-1)^{*}(1-(1-\exp (-$

alpha* $x^{\wedge}$ beta $\left.\left.\left.\left.\left.)\right)\right)^{\wedge}(b-1)^{*} \exp \left(\text { theta*(1-(1-(1-exp }\left(-a l p h a^{*} x^{\wedge} \text { beta }\right)^{\wedge} a\right)^{\wedge} b\right)\right)\right) /(\exp ($ theta $)-1)$

\}

cdfckwpd=function(x,par $)\{$

alpha=par[1]

beta=par[2]

theta=par[3]

$\mathrm{a}=\operatorname{par}[4]$

$\mathrm{b}=\operatorname{par}[5]$

$\left(\exp \left(\right.\right.$ theta*$\left.\left.\left(1-\left(1-\left(1-\exp \left(-a \text { alpha* } x^{\wedge} \text { beta }\right)^{\wedge} a\right)^{\wedge} b\right)\right)\right)-1\right) /(\exp ($ theta $)-1)$

\}

resultckwpd=goodness.fit $($ pdf=pdfckwpd,cdf=cdfckwpd,starts=c $(1,1,1,1,1)$, data=x, method="PSO",domain=c $(0$, Inf $)$, mle $=$ NULL,lim_inf $=c(0,0,0.1,0,0)$, lim_sup $=c(10,10,0.9,10,10))$

resultckwpd

pdfkwd=function(x,par) \{

alpha=par[1]

beta=par[2]

$\mathrm{a}=\operatorname{par}[3]$

$\mathrm{b}=\operatorname{par}[4]$

$a^{*} b^{*}$ alpha*beta* $x^{\wedge}\left(\right.$ beta-1)*exp(-alpha* $x^{\wedge}$ beta $) *\left(1-\exp \left(- \text { alpha* } x^{\wedge} \text { beta }\right)\right)^{\wedge}(a-1) *(1-(1-\exp (-$

alpha* $x^{\wedge}$ beta) $\left.)^{\wedge} a\right)^{\wedge}(b-1)$

\}

cdfkwd=function(x,par)\{

alpha=par[1]

beta=par[2] 
$\mathrm{a}=\operatorname{par}[3]$

$\mathrm{b}=\operatorname{par}[4]$

$1-\left(1-\left(1-\exp \left(- \text { alpha* } x^{\wedge} \text { beta }\right)\right)^{\wedge} a\right)^{\wedge} b$

\}

resultkwd=goodness.fit $(\mathrm{pdf}=\mathrm{pdfkwd}, \mathrm{cdf}=\mathrm{cdfkwd}, \mathrm{starts}=\mathrm{c}(1,1,1,1)$, data=x, method="PSO",domain=c(0,Inf $), \mathrm{m}$ le=NULL,lim_inf $=c(0,0,0,0), \lim \_$sup $\left.=c(10,10,10,10)\right)$

resultkwd

pdfkmwd=function(x,par) \{

alpha=par[1]

beta $=\operatorname{par}[2]$

theta=par[3]

$\mathrm{a}=\operatorname{par}[4]$

$\mathrm{b}=\operatorname{par}[5]$

$\mathrm{a}^{*} \mathrm{~b} *$ alpha* $\mathrm{x}^{\wedge}\left(\right.$ beta-1)*(beta+theta*x)*exp $\left(\text { theta* } \mathrm{x}-\text { alpha* } \mathrm{x}^{\wedge} \mathrm{beta}{ }^{*} \exp (\text { theta*} \mathrm{x})\right)^{*}(1-\exp (-$

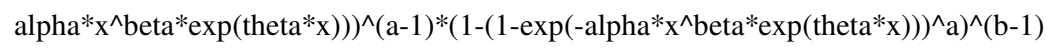

\}

cdfkmwd=function $(\mathrm{x}, \mathrm{par})\{$

alpha=par[1]

+ beta=par[2]

+ theta $=$ par[3]

$+\mathrm{a}=\operatorname{par}[4]$

$+b=\operatorname{par}[5]$

$+1-\left(1-\left(1-\exp \left(- \text { alpha* } x^{\wedge} \text { beta*exp }(\text { theta*x } x)\right)\right)^{\wedge} a\right)^{\wedge} b$

$+\}$

$>$

resultkmwd=goodness.fit $($ pdf=pdfkmwd,cdf=cdfkmwd,starts=c $(1,1,1,1,1)$, data=x, method="PSO",domain=c( 0$, Inf $),$ mle $=$ NULL,lim_inf $\left.=c(0,0,0,0,0), \lim \_\sup =\mathrm{c}(10,10,10,10,10)\right)$

$>$ resultkmwd

$>$ pdfkewd=function $(x$, par $)\{$ 
+ alpha=par[1]

+ beta=par[2]

+ theta=par[3]

$+\mathrm{a}=\operatorname{par}[4]$

$+b=\operatorname{par}[5]$

$+\quad \mathrm{a} * \mathrm{~b} *\left(\right.$ theta+beta*alpha* $\mathrm{x}^{\wedge}($ beta- 1$\left.)\right) * \exp \left(-\left(\text { theta*x+alpha* } \mathrm{x}^{\wedge} \text { beta }\right)\right)^{*}\left(1-\exp \left(-\left(\text { theta* } \mathrm{x}+\text { alpha* } \mathrm{x}^{\wedge} \text { beta }\right)\right)\right)^{\wedge}(\mathrm{a}-$

$1)^{*}\left(1-\left(1-\exp \left(-\left(\text { theta }^{*} x+\text { alpha* } x^{\wedge} \text { beta }\right)\right)\right)^{\wedge} a\right)^{\wedge}(b-1)$

$+\}$

$>$ cdfkewd=function $(\mathrm{x}$, par $)\{$

+ alpha=par[1]

+ beta=par[2]

+ theta $=\operatorname{par}[3]$

$+\mathrm{a}=\operatorname{par}[4]$

$+b=\operatorname{par}[5]$

$+1-\left(1-\left(1-\exp \left(-\left(\text { theta }^{*} x+\text { alpha* } x^{\wedge} \text { beta }\right)\right)\right)^{\wedge} a\right)^{\wedge} b$

$+\}$

$>$

resultkewd=goodness.fit $($ pdf=pdfkewd,cdf=cdfkewd,starts=c $(1,1,1,1,1)$, data=x, method="PSO",domain=c $(0, I$ nf),mle=NULL,lim_inf $\left.=\mathrm{c}(0,0,0,0,0), \lim \_\sup =\mathrm{c}(10,10,10,10,10)\right)$

$>$ resultkewd

$>$ pdfkwpd=function $(x, p a r)\{$

+ alpha=par[1]

+ beta=par[2]

+ theta $=\operatorname{par}[3]$

$+\mathrm{a}=\operatorname{par}[4]$

$+b=\operatorname{par}[5]$

$+\quad a^{*} b^{*}$ alpha*beta*theta* $x^{\wedge}\left(\text { beta-1)*exp(-alpha* } x^{\wedge} \text { beta }\right)^{*}\left(1-\exp \left(- \text { alpha* }{ }^{\wedge}{ }^{\wedge} \text { beta }\right)\right)^{\wedge}(a-1)^{*}(1-(1-\exp (-$ alpha* $x^{\wedge}$ beta $\left.\left.)\right)^{\wedge} a\right)^{\wedge}(b-1)^{*} \exp \left(- \text { theta*}^{*}\left(1-\left(1-\left(1-\exp \left(- \text { alpha* } x^{\wedge} \text { beta }\right)\right)^{\wedge} a\right)^{\wedge} b\right)\right)^{*}(1-\exp (- \text { theta }))^{\wedge}(-1)$

$+\}$

$>$ cdfkwpd=function $(\mathrm{x}$, par $)\{$

+ alpha=par[1] 
+ beta $=\operatorname{par}[2]$

+ theta $=$ par[3]

$+\mathrm{a}=\operatorname{par}[4]$

$+\mathrm{b}=\operatorname{par}[5]$

$+\left(1-\exp \left(- \text { theta }^{*}\left(1-\left(1-\left(1-\exp \left(- \text { alpha* } \mathrm{x}^{\wedge} \text { beta }\right)\right)^{\wedge} \mathrm{a}\right)^{\wedge} \mathrm{b}\right)\right)\right)^{*}(1-\exp (- \text { theta }))^{\wedge}(-1)$

$+\}$

$>$

resultkwpd=goodness.fit $(\mathrm{pdf}=$ pdfkwpd,cdf=cdfkwpd,starts=c $(1,1,1,1,1)$,data=x,method="PSO",domain=c $(0, \mathrm{I}$ nf),mle=NULL,lim_inf $\left.=\mathrm{c}(0,0,0,0,0), \lim \_\sup =\mathrm{c}(10,10,10,10,10)\right)$

$>$ resultkwpd

\section{Appendix C}

The $\mathrm{R}$ function for estimated densities of the distribution and the probability plot.

pdfckwpd=function(x,alpha, beta,theta, a,b)(a*b*alpha*beta*theta* $x^{\wedge}\left(\right.$ beta-1)*exp(-alpha* $\mathrm{x}^{\wedge}$ beta)*(1-exp(alpha* $x^{\wedge}$ beta $\left.)\right)^{\wedge}(a-1)^{*}\left(1-\left(1-\exp \left(-a l p h{ }^{*} x^{\wedge} \text { beta }\right)\right)\right)^{\wedge}(b-1)^{*} \exp \left(\right.$ theta* $\left.\left(1-\left(1-\left(1-\exp \left(-a l p h a^{*} x^{\wedge} \text { beta }\right)^{\wedge} a\right)^{\wedge} b\right)\right)\right) /$ $(\exp ($ theta $)-1))$

cdfckwpd=function $\left(x, a l p h a\right.$, beta,theta,a,b) $\left(\left(\exp \left(\right.\right.\right.$ theta $\left.\left.^{*}\left(1-\left(1-\left(1-\exp \left(-a l p h a^{*} x^{\wedge} \text { beta }\right)^{\wedge} a\right)^{\wedge} b\right)\right)\right)-1\right) /(\exp ($ theta $\left.)-1)\right)$ pdfkwd=function $(x$, alpha,beta, $a, b)\left(a * b * a l p h a *\right.$ beta* $^{\wedge}\left(\right.$ beta-1)*exp(-alpha* $x^{\wedge}$ beta $) *(1-\exp (-$ alpha* $x^{\wedge}$ beta $\left.\left.)\right)^{\wedge}(a-1)^{*}\left(1-\left(1-\exp \left(- \text { alpha* } x^{\wedge} \text { beta }\right)\right)^{\wedge} a\right)^{\wedge}(b-1)\right)$

cdfkwd=function $\left.\left.\left(x, \text { alpha, beta,a,b)(1-(1-(1-exp }\left(- \text { alpha* } x^{\wedge} \text { beta }\right)\right)^{\wedge} a\right)^{\wedge} b\right)$

pdfkmwd=function $(x$, alpha, beta,theta, $a, b)\left(a * b * a l p h a * x^{\wedge}(\right.$ beta- 1$) *\left(\right.$ beta+theta*x)*exp $\left(\right.$ theta $^{*} \mathrm{x}-$ alpha* $\mathrm{x}^{\wedge}$ beta*exp $($ theta*x $\left.)\right)^{*}\left(1-\exp \left(- \text { alpha* } \mathrm{x}^{\wedge} \text { beta*exp(theta*x) }\right)\right)^{\wedge}(\mathrm{a}-1) *(1-(1-\exp (-$ alpha* $x^{\wedge}$ beta*exp(theta*x)) $\left.\left.)^{\wedge} a\right)^{\wedge}(b-1)\right)$

cdfkmwd=function $\left(x\right.$, alpha, beta,theta,a,b) $\left.\left(1-\left(1-\left(1-\exp \left(- \text { alpha* } x^{\wedge} \text { beta*exp(theta*x }\right)\right)\right)^{\wedge} a\right)^{\wedge} b\right)$

pdfkewd=function $(\mathrm{x}$, alpha, beta, theta, $a, b)\left(a^{*} b^{*}\left(\text { theta+beta*alpha* } \mathrm{x}^{\wedge}(\text { beta- } 1)\right)^{*} \exp (-\right.$ $\left(\right.$ theta* $^{*} \mathrm{x}+$ alpha* $\mathrm{x}^{\wedge}$ beta $\left.)\right)^{*}\left(1-\exp \left(-\left(\text { theta* } \mathrm{x}+\text { alpha* } \mathrm{x}^{\wedge} \text { beta }\right)\right)\right)^{\wedge}(\mathrm{a}-1)^{*}\left(1-\left(1-\exp \left(-\left(\text { theta*} \mathrm{x}+\text { alpha* } \mathrm{x}^{\wedge} \text { beta }\right)\right)\right)^{\wedge} \mathrm{a}\right)^{\wedge}(\mathrm{b}-$ 1))

cdfkewd=function $(x, a l p h a$, beta,theta, $a, b)\left(1-\left(1-\left(1-\exp \left(-\left(\text { theta*}^{*} x+a l p h a^{*} x^{\wedge} \text { beta }\right)\right)\right)^{\wedge} a\right)^{\wedge} b\right)$

pdfkwpd=function $(\mathrm{x}$, alpha, beta,theta, $a, b)\left(a * b * a l p h a * b e t a * \text { theta*x^(beta-1)*exp(-alpha* } \mathrm{x}^{\wedge} \text { beta }\right)^{*}(1-\exp (-$ alpha* $x^{\wedge}$ beta $\left.)\right)^{\wedge}(a-1)^{*}\left(1-\left(1-\exp \left(-a l p h a^{*} x^{\wedge} \text { beta }\right)\right)^{\wedge} a\right)^{\wedge}(b-1)^{*} \exp \left(-t_{h e t a *}\left(1-\left(1-\left(1-\exp \left(-a l p h a^{*} x^{\wedge} \text { beta }\right)\right)^{\wedge} a\right)^{\wedge} b\right)\right)^{*}(1-$ $\exp (-$ theta $\left.))^{\wedge}(-1)\right)$

cdfkwpd=function $\left(x\right.$, alpha,beta, theta,a,b) $\left(\left(1-\exp \left(- \text { theta }^{*}\left(1-\left(1-\left(1-\exp \left(- \text { alpha* } x^{\wedge} \text { beta }\right)\right)^{\wedge} a\right)^{\wedge} b\right)\right)\right)^{*}(1-\exp (-\right.$ theta) $\left.)^{\wedge}(-1)\right)$ 
$\mathrm{x}=\mathrm{c}(0.55,1.28,1.51,1.61,1.70,2.00,0.74,1.29,1.52,1.62,1.7,2.01,0.77,1.3,1.53,1.62,1.73,2.24,0.81,1.36,1.54,1$ $.63,1.76,0.84,1.39,1.55,1.55,1.64,1.76,0.93,1.42,1.55,1.66,1.77,1.04,1.48,1.58,1.66,1.78,1.11,1.48,1.59,1.66$ ,1.81,1.13,1.49,1.6,1.67,1.82,1.24,1.49,1.61,1.68,1.84,1.25,1.5,1.61,1.68,1.84,1.27,1.50,1.61,1.69,1.89) hist(x,prob=T,xlab="x-value",ylab="f(x)",main="Estimated Pdfs for strengths of $1.5 \mathrm{~cm}$ glass fibers") curve(pdfckwpd(x,1.4883772,1.1298867,0.8288365,1.4584510,1.2212270),add=T,lty=1,lwd=2,col="red") curve(pdfkwd(x,0.8605495,0.9278466,0.8633339,0.9021568),add=T,lty=1,lwd=2,col="blue") curve(pdfkmwd(x,0.5971527,0.5931218,0.5825983,0.5773604,0.5723678),add=T,lty=1,lwd=2,col="orange") curve(pdfkewd(x,0.2639996,0.2515058,0.2226657,0.2535793,0.2308934),add=T,lty=1,lwd=2,col="green") curve(pdfkwpd(x,0.6006863,0.5996338,0.5994494,0.5987368,0.5989666),add=T,lty=1,lwd=2,col="cyan") legend(“topright”,cex=0.8,title="key",c("CKWPD","KWD","KMWD","KEWD","KWPD"),bty="n",horiz=F ALSE, lty=c(1,1,1),lwd=c(2,2,2,2,2),col=c("red","blue","orange","green","cyan"))

plot(ecdf(x),xlab="x-value",ylab="F(x)",main="Estimated Cdfs for strengths of $1.5 \mathrm{~cm}$ glass fibers") curve(cdfckwpd(x,1.4883772,1.1298867,0.8288365,1.4584510,1.2212270),add=T,lty=1,lwd=2,col="red") curve(cdfkwd(x,0.8605495,0.9278466,0.8633339,0.9021568),add=T,lty=1,lwd=2,col="blue") curve(cdfkmwd(x,0.5971527,0.5931218,0.5825983,0.5773604,0.5723678),add=T,lty=1,lwd=2,col="orange") curve(cdfkewd(x,0.2639996,0.2515058,0.2226657,0.2535793,0.2308934),add=T,lty=1,lwd=2,col="green") curve(cdfkwpd(x,0.6006863,0.5996338,0.5994494,0.5987368,0.5989666),add=T,lty=1,lwd=2,col="cyan") legend(“topright”, cex=0.8,title="key",c("ECDF","CKWPD","KWD","KMWD","KEWD","KWPD"),bty="n", horiz=FALSE,lty=c(6,1,1,1,1,1),lwd=c(3,2,2,2,2,2),col=c("black","red","blue","orange","green","cyan")) $\mathrm{n}=$ length $(\mathrm{x})$

$\mathrm{y}=\operatorname{sort}(\mathrm{x})$

$\mathrm{z}=1: \mathrm{n}$

$\mathrm{a}=\mathrm{z} / \mathrm{n}$

$\mathrm{A}=\mathrm{cdfckwpd}(\mathrm{y}, 1.4883772,1.1298867,0.8288365,1.4584510,1.2212270)$

$\mathrm{B}=\mathrm{cdfkwd}(\mathrm{y}, 0.8605495,0.9278466,0.8633339,0.9021568)$

$\mathrm{C}=\mathrm{cdfkmwd}(\mathrm{y}, 0.5971527,0.5931218,0.5825983,0.5773604,0.5723678)$

$\mathrm{D}=\mathrm{cdfkmwd}(\mathrm{y}, 0.5971527,0.5931218,0.5825983,0.5773604,0.5723678)$

$\mathrm{E}=$ cdfkwpd $(\mathrm{y}, 0.6006863,0.5996338,0.5994494,0.5987368,0.5989666)$

qqplot(a,A,xlab="Observed Probability", ylab="Expected Probability", main="QQ-Plot for CKWPD")

abline $(0,1, \mathrm{col}=" \mathrm{red} ")$

$\underline{\text { qqplot(a,B,xlab="Observed Probability", ylab="Expected Probability", main="QQ-Plot for KWD") }}$ 
abline $(0,1$, col="blue")

qqplot(a,C,xlab="Observed Probability", ylab="Expected Probability", main="QQ-Plot for KMWD")

abline $(0,1$, col="orange")

qqplot(a,D,xlab="Observed Probability", ylab="Expected Probability", main="QQ-Plot for KEWD")

abline $(0,1$, col="green")

qqplot(a,E,xlab="Observed Probability", ylab="Expected Probability", main="QQ-Plot for KWPD")

abline $(0,1$, col="cyan")

Innocent Boyle Eraikhuemen

Department of Physical Sciences, Benson Idahosa University, Benin City, Edo State, Nigeria

e-mail: ieraikhuemen@biu.edu.ng

Julian Ibezimako Mbegbu and Friday Ewere

Department of Statistics, University of Benin, Benin City, Edo State, Nigeria

e-mail: Julian.mbegbu@uniben.edu

Friday.ewere@uniben.edu

This is an open access article distributed under the terms of the Creative Commons Attribution License (http://creativecommons.org/licenses/by/4.0/), which permits unrestricted, use, distribution and reproduction in any medium, or format for any purpose, even commercially provided the work is properly cited.. 